\title{
A Baseline Load Schedule for the Manual Calibration of a Force Balance
}

\author{
N. Ulbrich* \\ Jacobs Technology Inc., Moffett Field, California 94035-1000 \\ R. Gisler** \\ NASA Ames Research Center, Moffett Field, California 94035-1000
}

\begin{abstract}
A baseline load schedule for the manual calibration of a force balance was developed that takes current capabilities at the NASA Ames Balance Calibration Laboratory into account. The load schedule consists of 18 load series with a total of 194 data points. It was designed to satisfy six requirements: (i) positive and negative loadings should be applied for each load component; (ii) at least three loadings should be applied between $0 \%$ and $100 \%$ load capacity; (iii) normal and side force loadings should be applied at the forward gage location, the aft gage location, and the balance moment center; (iv) the balance should be used in UP and DOWN orientation to get axial force loadings; (v) the constant normal and side force approaches should be used to get the rolling moment loadings; (vi) rolling moment loadings should be obtained for $0,90,180$, and 270 degrees balance orientation. Three different approaches are also reviewed that may be used to independently estimate the natural zeros of the balance. These three approaches provide gage output differences that may be used to estimate the weight of both the metric and non-metric part of the balance. Manual calibration data of NASA's MK29A balance and machine calibration data of NASA's MC60D balance are used to illustrate and evaluate different aspects of the proposed baseline load schedule design.
\end{abstract}

\section{Nomenclature}

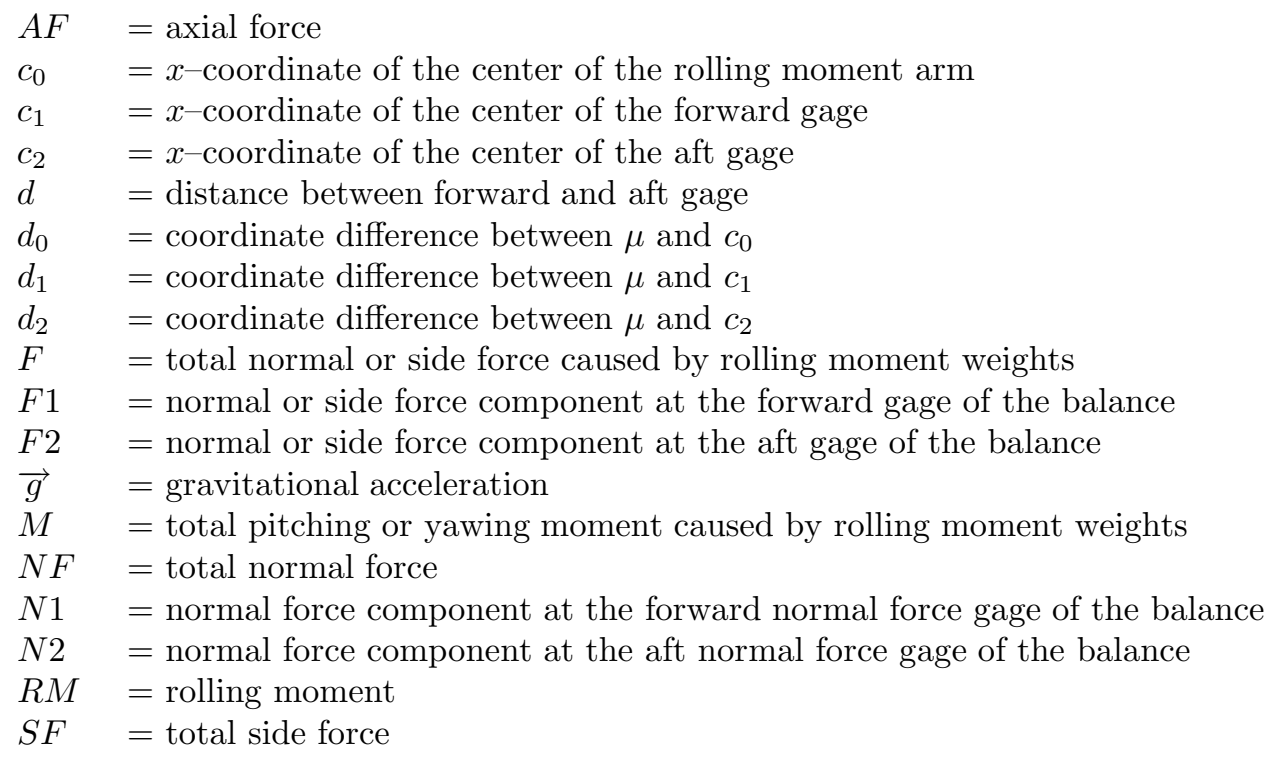

* Aerodynamicist, Jacobs Technology Inc.

** Instrumentation Engineer, Code AOI, NASA Ames Research Center 
$S 1 \quad=$ side force component at the forward side force gage of the balance

$S 2 \quad=$ side force component at the aft side force gage of the balance

$x \quad=$ coordinate along the roll axis of the balance

$\mu \quad=x$-coordinate of the balance moment center

\section{Introduction}

A baseline load schedule for the manual calibration of a force balance was developed at the NASA Ames Balance Calibration Laboratory. The proposed load schedule was designed to best capture the physical behavior of a force balance while taking limitations of (i) the manual calibration process and of (ii) the calibration hardware at the Ames Balance Calibration Laboratory into account.

The baseline load schedule consists of 18 load series with a total of 194 data points. The load schedule assumes that gravity weights are used to apply the loadings. Therefore, the balance orientation for each load series must be specified. The balance orientation essentially describes the location of the roll, pitch, and yaw axes of the balance relative to the gravitational acceleration. Figure 1a shows the six distinct orientations that the baseline load schedule uses.

In general, the baseline load schedule satisfies six requirements: (1) Positive and negative loads are applied for each load component so that the symmetry/asymmetry of the balance behavior and its "bidirectionality" characteristics are captured. (2) At least three loadings are applied between $0 \%$ and $100 \%$ load capacity so that a better definition of the quadratic terms in the regression model of the balance calibration data is achieved. (3) Normal and side forces are applied at the forward gage location, the aft gage location, and the balance moment center so that relationships between the location of the applied forces and the location of the strain-gage measurements are described. (4) The UP and DOWN balance orientation is used to apply positive and negative axial force loadings as the direction of the gravitational acceleration may be used for the alignment of axial forces. (5) The "constant" normal and side force approaches are used to get rolling moment loadings as the balance may not have to be re-leveled whenever a rolling moment loading is changed by shifting gravity weights from weight pan to weight pan. (6) Rolling moment loadings are performed for $0,90,180$, and 270 degrees balance orientation in order to capture the symmetry/asymmetry of the balance behavior. The table in Fig. 1b summarizes features of the baseline load schedule.

The natural zeros, i.e., the gage outputs of the absolute load datum of the balance, also have to be obtained during the calibration. These outputs are an important "universal" reference of a strain-gage balance. In addition, the natural zeros are needed whenever a balance calibration data analysis requires a tare load iteration (see Refs. [1] and [2] for a discussion of the tare load iteration process). The Ames Balance Calibration Laboratory supports three independent methods to determine the natural zeros. These three approaches do not just provide outputs for the calculation of the absolute load datum of the balance. They can also be used to numerically assess the weight of both the metric and non-metric part of the balance. This information may be used to validate results of the tare load iteration process as the sum of the estimated weights of the metric and non-metric parts may be compared with the known total weight of the balance.

Key elements of the proposed load schedule are discussed in the next part of the paper. Then, the three independent methods are reviewed that may be used to determine the natural zeros. Finally, data from a manual calibration of the NASA's MK29A balance and data from a machine calibration of NASA's MC60D balance are used to evaluate the proposed baseline load schedule.

\section{Baseline Load Schedule}

\section{A. General Remarks}

The baseline load schedule defined in Fig. 1b assumes that the gravity weight inventory of a balance calibration laboratory will allow for the application of at least three loadings between $0 \%$ and $100 \%$ load capacity. For simplicity, "ideal" load levels are used in Fig. 1b to describe sets of loadings that fulfill this requirement (i.e., $0 \%, \pm 25 \%, \pm 50 \%, \cdots$ ). Individual loads should be applied in a gradually increasing/decreasing fashion using more or less equally spaced increments from $0 \%$ to $100 \%$ capacity. A balance calibration laboratory may only have specific sets of gravity weights in its inventory that may not allow for 
an even spacing between $0 \%$ and $\pm 100 \%$ load capacity. Then, the "ideal" load levels listed in Fig. 1b should be approximated as closely as possible by the available weights.

Calibration load series 3, 6, 9, and 12 of the baseline load schedule apply loadings at the balance moment center (BMC). In those four cases a loading of $\pm 100 \%$ of capacity means that the corresponding load components at the forward and aft gages of the balance are simultaneously loaded to $\pm 100 \%$ of capacity.

Repeat points are also a part of each load series that is listed in Fig. 1b. Repeat points approach an already existing load of a load series by removing instead of adding weights (or vice versa). Therefore, repeat points help characterize the hysteresis behavior of the balance.

\section{B. Normal Force Components}

Six load series with a total of 54 calibration points are needed to apply the normal force loadings. The 54 calibration points consist of 30 unique data points and 24 repeat points. Figures $2 \mathrm{a}$ and $2 \mathrm{~b}$ show a typical setup of a force balance before and during the application of gravity weights whenever normal force components are applied. Load series 1, 2, and 3 apply gravity weights such that the positive normal force is in the direction of the gravitational acceleration (orientation $\equiv 0$ ). Load series 4,5 , and 6 apply gravity weights such that the negative normal force is in the direction of the gravitational acceleration (orientation $\equiv 180$ ). The individual loadings are applied at three locations on the calibration body: forward gage, aft gage, and BMC.

\section{Side Force Components}

The application of side force loadings is very similar to the application of normal force loadings. Again, six load series with a total of 54 calibration points are needed to apply the side force loadings. The 54 calibration points consist of 30 unique data points and 24 repeat points.

Figures $2 \mathrm{a}$ and $2 \mathrm{~b}$ show a typical setup of a force balance before and during the application of gravity weights whenever side force components are applied. Load series 7, 8, and 9 apply gravity weights such that the positive side force is in the direction of the gravitational acceleration (orientation $\equiv 90$ ). Load series 10,11 , and 12 apply gravity weights such that the negative side force is in the direction of the gravitational acceleration (orientation $\equiv 270$ ). Again, as it was the case with the normal force loadings, the individual loads are applied at three locations on the calibration body: forward gage, aft gage, and BMC.

\section{Rolling Moment with Constant Normal and Side Force}

Four load series with a total of 68 calibration points are needed to apply the rolling moment loadings at constant total normal and side force. These 68 calibration points consist of 36 unique data points and 32 repeat points.

The application of rolling moment loadings starts by first attaching the rolling moment hardware to the calibration body such that the rolling moment arm is perpendicular to (i) the direction of the normal force and (ii) the direction of the roll axis of the balance. Then, the balance is aligned such that the positive total normal force $N F$ points in the direction of the gravitational acceleration (orientation $\equiv 0$ ). In the next step, loadings at constant total normal force $N F$ are applied. This approach has the advantage that a single independent variable, i.e., the rolling moment, is changed during the calibration experiment while simultaneously keeping all other balance forces and moments constant. In addition, the balance needs to be leveled only once during the entire load series assuming that small alignment corrections are ignored. This is possible because balance deflections remain more or less constant as long as (i) the total normal force does not change and (ii) the balance support is symmetric relative to the plane that is defined by the roll axis of the balance and the direction of the gravitational acceleration. An equal number of gravity weights is placed on each of the two weight pans at the ends of the rolling moment arm to establish a zero rolling moment at constant normal force. Weights are then simply shifted from one weight pan to another to get the desired rolling moment loadings. This situation is described in load series 13 (see Fig. 1b).

In the next step, the balance is rotated such that the negative total normal force $N F$ is in the direction of the gravitational acceleration (orientation $\equiv 180$ ). Then, the balance is leveled and the loadings of load series 14 are applied (see Fig. 1b). Load series 14 is the mirror image of load series 13 for negative total normal force $N F$.

The rolling moments for constant total side force $S F$ need to be applied next. Now, the rolling moment hardware is attached to the calibration body such that the rolling moment arm is perpendicular to (i) the 
direction of the side force and (ii) the direction of the roll axis of the balance. Then, the balance is aligned such that the positive total side force $S F$ points in the direction of the gravitational acceleration (orientation $\equiv 90$ ). In the next step, the loadings at constant total side force $S F$ are applied. An equal number of weights is placed on each of the two weight pans at the ends of the rolling moment arm to establish a zero rolling moment at constant side force. Weights are then shifted from one weight pan to another and back to get the desired rolling moment loadings. This situation is described in load series 15 (see Fig. 1b).

Now, the balance is rotated such that the negative total side force $S F$ is in the direction of the gravitational acceleration (orientation $\equiv 270$ ). Then, the balance is leveled and the loadings of load series 16 are applied (see Fig. 1b). Load series 16 is the mirror image of load series 15 for negative total side force $S F$.

It is important to point out that all rolling moment loadings of load series 13, 14, 15, and 16 are "combined" loadings as the forward and aft normal (or side force) components are loaded at the same time. The total normal force $N F$ (or the total side force $S F$ ) is the force $F$ that is caused by the gravity weights. Therefore, relationships between the "known" total force $F$ and the "unknown" force components $F 1$ and $F 2$ at the forward and aft gages have been derived in the appendix of the paper so that the final balance calibration load table can be prepared in the correct format (see Eqs. (11a) and (11b) in the appendix).

Calibration hardware limitations or other practical constraints may not always allow for the application of all four sets of rolling moment loadings that the baseline load schedule suggests (i.e., load series 13, 14, 15, and 16). It may also be sufficient for some balances to only use the normal (or side) force components for the description of the electrical outputs caused by an applied rolling moment. In these cases an attempt should be made at least to apply the rolling moment loadings for $\pm N F$ (or $\pm S F$ ) so that the symmetry/asymmetry between rolling moment gage outputs and rolling moment loadings can be characterized.

\section{E. Axial Force}

Two load series with a total of 18 calibration points are needed to apply the axial force loadings. The 18 calibration points consist of 10 actual data points and 8 repeat points.

Figures $3 \mathrm{a}$ and $3 \mathrm{~b}$ show a typical setup of a force balance before and during the application of gravity weights whenever axial force loadings are applied at the Ames Balance Calibration Laboratory. The axial force and the gravitational acceleration are parallel to each other. This situation greatly simplifies the alignment of the axial force relative to the balance roll axis $(x$-axis). In addition, knife edges are used to establish a contact line between calibration body and yoke assembly.

Load series 17 applies gravity weights such that the positive axial force points in the direction of the gravitational acceleration (orientation $\equiv$ UP). Load series 18 applies gravity weights such that the negative axial force points in the direction of the gravitational acceleration (orientation $\equiv$ DOWN).

It may not always be possible to align the balance roll axis parallel to the direction of the gravitational acceleration. Some balances may require the application of axial loadings while the balance roll axis is perpendicular to the gravitational acceleration. This often complicates the alignment of the axial loadings because the direction of the gravitational acceleration can no longer directly be used for the alignment.

The natural zeros of the balance also need to be determined so that the balance behavior can be described correctly. Different options exist in order to determine the natural zeros. They are explained in detail in the next section of the paper.

\section{Natural Zeros}

Data points need to be recorded during the manual calibration of a force balance that make it possible to determine the gage outputs of the absolute load datum of the balance. These gage outputs are called the "natural zeros" of the balance. "Natural zeros" are also measured as a part of the standard electrical checks on the balance. An unexpected shift of the "natural zeros" may indicate a possible damage of the balance since it was last used.

The absolute load datum is assumed to be a zero load condition of the balance. Corresponding gage outputs, i.e., the "natural zeros," would be obtained if the balance is in a "weightless" condition (see Fig. 4a). Unfortunately, it is not directly possible to put a balance into "weightless" state in a balance calibration laboratory. Instead, outputs of a "weightless" balance have to be approximated by using the arithmetic mean of outputs that are measured for different orientations of the balance. 
In principle, three independent methods are available to determine the natural zeros of a balance (Method A, Method B, Method C). The table in Fig. 4b compares characteristics of these three methods. All methods determine the natural zeros by averaging gage outputs that are measured for sets of specific balance orientation combinations. The orientation combinations are subsets of the six basic orientations that are defined in Fig. 1a.

Orientations listed in Fig. 4b are slightly modified versions of the original orientation identifiers that are defined in Fig. 1a. They have an extension that is either $-S$ or $-C$. The extension $-S$ indicates that the acting balance load is caused by the weight of the balance shell (metric part). Similarly, the extension $-C$ indicates that the acting balance load is caused by the weight of the balance core (non-metric part).

Figure 5a shows the setup of a balance assuming that Method A is applied. In this case, the roll axis of the balance is perpendicular to the gravity vector. The balance is rotated four times $(0,90,180,270$ degrees $)$ and corresponding gage outputs are recorded. These gage outputs are caused by the weight of the shell of the balance, i.e., by the weight of the metric part, because the non-metric part of the balance is supported. Then, the arithmetic mean of the outputs is computed for each gage. These values are the first estimate of the natural zeros of the balance.

Sometimes, a better horizontal alignment of the balance can be achieved by keeping the calibration body attached to the balance (see Fig. $5 \mathrm{~b}$ ). In that case, the rotation of the balance and the calculation of the natural zeros is still performed as outlined in the previous paragraph. The influence of the calibration body weight on the natural zeros is removed by the averaging of the gage outputs of the four orientations. However, the gage outputs for each balance orientation are now caused by the shell assembly weight, i.e., the combined weight of the calibration body and the metric part, assuming that the weight of attached alignment sensors is considered to be a part of the weight of the calibration body.

Figure 6a shows the setup of a balance assuming that Method B is applied. In this case, the roll axis of the balance is parallel to the gravity vector. The balance is rotated two times (orientations UP and DOWN) and corresponding gage outputs are recorded. Again, gage outputs are caused by the weight of the shell of the balance, i.e., by the weight of the metric part. Then, the arithmetic mean of the outputs is computed for each gage. These values are the second independent estimate of the natural zeros of the balance.

A better vertical alignment of the balance can often be achieved by keeping the calibration body attached to the balance (see Fig. 6b). In that case, the rotation of the balance and the calculation of the natural zeros is still performed as outlined in the previous paragraph as the influence of the calibration body weight on the gage outputs is removed by the averaging of the outputs of each gage.

Figure 7a shows the setup of a balance assuming that Method $\mathrm{C}$ is applied. In this case, the roll axis of the balance is perpendicular to the gravity vector as the balance is assumed to be placed on a leveling table using a vee block. Again, the balance is rotated four times (0, 90, 180, 270 degrees) and corresponding gage outputs are recorded. Now, the gage outputs of each orientation are caused by the weight of the core of the balance, i.e., by the weight of the non-metric part, because the metric part of the balance is support. Then, the arithmetic mean of the outputs is computed for each gage. These values are the third estimate of the natural zeros of the balance. Method C may also be repeated with the calibration body left attached to the balance (see Fig. 7b). In theory, the gage outputs measured for the situations depicted in Figs. 7a and 7b should be very close as the gages only see loads caused by the balance core (non-metric part).

Experience at the Ames Balance Calibration Laboratory has shown that Method A, Method B, and Method C will lead to virtually the same natural zero estimates as long as small balance alignment and gage output measurement errors are neglected. Ultimately, the choice of methods is determined in part by the equipment available at the time of the calibration. The preferred method at Ames is Method C with calibration body attached to insure accurate horizontal/vertical alignment and rotations.

\section{Discussion of Example}

Data from the manual calibration of NASA's MK29A balance is used in this section to illustrate and evaluate different characteristics of the proposed baseline load schedule. The Ames MK29A balance was manufactured by the Task Corporation. It is a six-component force balance that measures five forces and one moment $(N 1, N 2, S 1, S 2, A F, R M)$. It has a diameter of 2.0 inches and a total length of 11.25 inches. Table 1 below shows the capacity of each load component of the MK29A balance. 
Table 1: Load capacities of the MK29A balance.

\begin{tabular}{|c|c|c|c|c|c|c|}
\hline & N1, lbs & N2, lbs & S1, lbs & S2, lbs & RM, in-lbs & AF, lbs \\
\hline Capacity & 2100 & 2100 & 700 & 700 & 3800 & 350 \\
\hline
\end{tabular}

The balance calibration was performed using a total of 157 manual loadings. The loadings were applied in 16 load series that tried to best follow the proposed baseline load schedule. Load series 15 and 16 of the baseline load schedule were removed from the calibration data set because of data quality issues. In addition, due to calibration hardware limitations, the normal and side force loadings at the BMC were only performed up to about half of gage capacity.

Figure 8 shows the final set of loadings that were performed after tare corrections for the weight of the calibration equipment were applied to the original load set using a tare load iteration scheme that is described in Ref. [2]. It can be seen in Fig. 8 that most load series were performed as a series of single gage loadings, i.e., as a series of loadings that primarily caused outputs on a single gage. The loadings of series $3,6,9,12,13$, and 14 , on the other hand, are combined loadings. The loadings of series $3,6,9$, and 12 are normal or side force loadings that were applied at the BMC of the balance. The loadings of series 13 and 14, on the other hand, are rolling moment loadings. In those cases, positive and negative rolling moments were applied while keeping the total normal force constant.

Figure 9a shows the gage outputs of the natural zero points that were used to compute the natural zeros

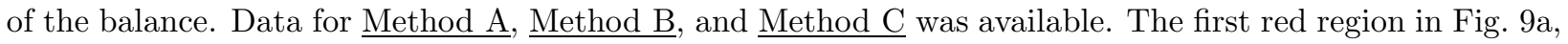
for example, shows the outputs of the natural zero points that Method A needs. The second red region shows the corresponding averaged outputs. They are a first estimate of the natural zeros of the balance. Similar estimates may be obtained by using the data for Method B and Method C. Finally, the green region in Fig. 9a shows the natural zeros that are obtained after averaging the independent natural zero estimates of Method A, Method B, and Method C.

The weights of the balance shell (metric part) and core (non-metric part) may be obtained by processing the gage outputs of the 10 natural zero points that are listed in Fig. 9a. First, the difference between these gage outputs and the final natural zeros of the balance, i.e., the values of the green region in Fig. 9a, are computed (these differences are listed in the first table of Fig. 9b). Then, the differences are used as input for the load iteration scheme that may be used in combination with the Iterative Method for the calculation of balance loads (see Ref. [1] for a description of the Iterative Method). The result of these load calculations are the loads that are given in the second table of Fig. 9b. The loads highlighted in blue color are forces that are related to the balance shell and core weights. Table 2 below lists corresponding balance component weights that can be obtained from forces highlighted in blue color in Fig. 9b.

Table 2: Summary of estimated balance component weights of the MK29A balance.

\begin{tabular}{|c|c|c|c|c|}
\hline Index & Ident. & Orient. & $\begin{array}{c}\text { Acting Balance Load } \\
\text { (shell } \equiv \text { metric part } ; \text { core } \equiv \text { non-metric part) }\end{array}$ & Weight, lbs \\
\hline 1 & NZ-0001 & $0-\mathrm{S}$ & shell weight $=|N 1+N 2|$ & 2.75 \\
\hline 2 & NZ-0002 & $90-\mathrm{S}$ & shell weight $=|S 1+S 2|$ & 2.68 \\
\hline 3 & NZ-0003 & $180-\mathrm{S}$ & shell weight $=|N 1+N 2|$ & 2.10 \\
\hline 4 & NZ-0004 & $270-\mathrm{S}$ & shell weight $=|S 1+S 2|$ & 2.67 \\
\hline 5 & NZ-0005 & UP-S & shell weight $=|A F|$ & 2.92 \\
\hline 6 & NZ-0006 & DOWN-S & shell weight $=|A F|$ & 3.23 \\
\hline 7 & NZ-0007 & $0-\mathrm{C}$ & core weight $=|N 1+N 2|$ & 3.98 \\
\hline 8 & NZ-0008 & $90-\mathrm{C}$ & core weight $=|S 1+S 2|$ & 3.45 \\
\hline 9 & NZ-0009 & $180-\mathrm{C}$ & core weight $=|N 1+N 2|$ & core weight $=|S 1+S 2|$ \\
\hline 10 & NZ-0010 & $270-\mathrm{C}$ & & \\
\hline
\end{tabular}

American Institute of Aeronautics and Astronautics 
The weights of indices 1 to 6 in Table 2 are the individual shell weight estimates. The mean of the estimated values is $2.65[l b s]$. Similarly, the weights of indices 7 to 10 in Table 2 are the individual core weight estimates. The mean of the estimated values is $3.80[\mathrm{lbs}]$. The sum of the estimated shell and core weights is a numerical approximation of the total weight of the balance. It is estimated to be $6.45[\mathrm{lbs}]$.

A precision scale was used to weigh the balance so that the accuracy of the numerical approximation of the balance weight could be assessed. The direct measurement of the balance weight was performed such that the weight of the balance cable would be excluded. The precision scale determined the weight of the balance to be $6.74[l b s]( \pm 0.05[l b s])$. This value is very close to the numerical approximation of $6.45[l b s]$ that was obtained above from the gage outputs measurements of the 10 natural zero points (the actual difference between measured and approximated value is $\approx 0.29[l b s]$ ). The excellent agreement between measured and approximated value validates the approach that is used at the Ames Balance Calibration Laboratory for the indirect determination of the weight of the balance shell. This information is needed whenever results of the tare load iteration process have to be tested for accuracy as the weight of the balance shell is the only significant part of the tare load estimate that cannot directly be measured (see, e.g., Ref. [1] for a description of basic elements of the tare load iteration process that is used at the Ames Balance Calibration Laboratory).

Figure 10 show the symmetry in the combined load plot of $N 1$ and $R M$ that results from applying load series 13 and 14 . The small constant positive/negative offsets of the $N 1$ load component in the $N 1$ axis direction are the tare weights that are caused by the calibration fixtures and the gravity weights.

Figure 11 shows where the side force loads at the BMC appear in the combined load plot of the two side force components $S 1$ and $S 2$. They are on the principle diagonals of the first and third quadrant. The loadings of the MK29A balance at the BMC did only extend to about half of the capacity of the side force gages (the missing loadings are highlighted using green dashed lines in Fig. 11). Therefore, not all loads of the baseline load schedule were applied that may be needed for a good mathematical description of the balance behavior.

\section{Assessment of Predictive Capability}

Data from a machine calibration of NASA's MC60D balance is used in this section for a preliminary assessment of the accuracy of regression models and data reduction matrices that are derived from the proposed baseline load schedule. The use of this 1906-point machine calibration data set for the accuracy study became possible because it contained a 142-point subset of calibration points that closely matched the proposed baseline load schedule.

The MC60D balance used for the accuracy assessment needs to be described in more detail. It is a six-component force balance that was manufactured by Triumph Aerospace in San Diego. It measures five forces and one moment $(N 1, N 2, S 1, S 2, A F, R M)$ and has a diameter of 2.0 inches. Table 3 below lists load capacities of the balance:

Table 3: Load capacities of the MC60D balance.

\begin{tabular}{|l|c|c|c|c|c|c|}
\hline & N1, lbs & N2, lbs & S1, lbs & S2, lbs & RM, in-lbs & AF, lbs \\
\hline Capacity & 2500 & 2500 & 1250 & 1250 & 5000 & 700 \\
\hline
\end{tabular}

Data from the machine calibration of the MC60D balance was made available for the current study. The original machine calibration consists of 1906 data points that were obtained in Triumph's ABCS balance calibration machine. During a detailed inspection of the machine calibration data of the MC60D it was noticed that a subset of 142 data points could be extracted from the original data set that closely matches the baseline load schedule proposed in the present paper. Consequently, this 142-point subset of the machine calibration is equivalent to a manual calibration data set of the balance. Then, a data reduction matrix could be derived from the 142-point subset that could be tested for accuracy by using the original 1906-point machine calibration data set as precision check loads.

Figure 12 shows the load schedule of the 142-point subset of the original 1906-point machine calibration data of the MC60D balance. The following observations can be made after comparing the load schedule of the MC60D (Fig. 12) with the corresponding load schedule of the MK29A (Fig. 8): (i) The loadings at the 
forward and aft normal/side force gages, i.e., series 1, 2, 4, 5, 7, 8, 10, 11, for the MK29A and the MC60D are very similar; (ii) the normal/side forces at the BMC, i.e., series $3,6,9,12$, are significantly larger than $50 \%$ of capacity for the MC60D balance; (iii) The rolling moment series of the MC60D data set, i.e., series 13 , is not a combined loading as both the normal and side force components are zero; (iv) The axial force series of the MC60D balance, i.e., series 17 and 18, are symmetric.

It was decided to derive two data reduction matrices in order to perform the evaluation of the baseline load schedule approximation that is defined by the 142-point subset. The first matrix, i.e., matrix 1, was computed by using the 142 -point subset as input. The second matrix, i.e., matrix 2 , was computed by using the original 1906-point data set as input. Regression models required for the calculation of the two matrices were obtained by applying a regression model optimization algorithm to the corresponding data sets that was developed at NASA Ames Research Center (see Ref. [3] for a description of an earlier version of the optimization algorithm).

Figure 13a shows the load residuals of the 142-point subset after the data reduction matrix derived from the 142-point subset, i.e., matrix 1, was used for the prediction of loads. Figure 13b shows the load residuals of the original 1906-point data set after the data reduction matrix derived from the original 1906-point data set, i.e., matrix 2 , was used for the prediction of loads. Figure 13c shows the load residuals of the original 1906-point data set after the data reduction matrix derived from the 142-point subset, i.e., matrix 1, was used for the prediction of loads. Table 4 below summarizes standard deviations of the load residuals for the three cases that are depicted in Figs. 13a, 13b, and 13c.

Table 4: Standard deviation of load residuals in percent of load capacity.

\begin{tabular}{|c|c|c|c|c|c|c|}
\hline Load Residual Set Description & $\mathrm{N} 1, \%$ & $\mathrm{~N} 2, \%$ & $\mathrm{~S} 1, \%$ & $\mathrm{~S} 2, \%$ & $\mathrm{RM}, \%$ & $\mathrm{AF}, \%$ \\
\hline $\begin{array}{l}\text { Figure 13a: residuals of } 142-\text { point subset of machine calibration } \\
\text { after applying matrix of } 142-\text { point subset }(\underline{\text { matrix } 1})\end{array}$ & 0.0497 & 0.0741 & 0.1466 & 0.1240 & 0.0588 & 0.0431 \\
\hline $\begin{array}{l}\text { Figure 13b: residuals of } 1906-\text { point machine calibration after } \\
\text { applying matrix of } 1906-\text { point machine calibration ( } \underline{\text { matrix } 2} \text { ) }\end{array}$ & 0.0658 & 0.0942 & 0.1944 & 0.1814 & 0.1439 & 0.0621 \\
\hline $\begin{array}{l}\text { Figure 13c: residuals of } 1906-\text { point machine calibration } \\
\text { after applying matrix of } 142-\text { point subset ( } \text { matrix } 1)\end{array}$ & 0.1257 & 0.3511 & 0.3297 & 0.4822 & 0.1990 & 0.3591 \\
\hline
\end{tabular}

The green regions in Fig. 13c mark the residual range that was obtained by using matrix 2, i.e., the matrix obtained from the original 1906-point data set (see also Fig. 13b). The red regions in Fig. 13c mark the residual increases caused by applying matrix 1, i.e., the matrix obtained from the 142-point subset, to the original 1906-point data set. It can be seen that the use of matrix 1, i.e., the matrix obtained from the 142-point subset, greatly increases the load residuals whenever complex loadings are applied to the balance that cause noticable interactions between the gages.

The comparison of the three cases listed in Table 4 clearly shows limitations of the baseline load schedule as far as the prediction of balance loads is concerned. The matrix derived from the baseline load schedule performs well as long as a balance experiences loadings that are similar to the ones applied during the execution of the baseline load schedule (cf. Fig. 13a). The matrix, however, does not perform well if the balance experiences combined loadings and gage interactions that the balance did not see during the application of the baseline load schedule (cf. Fig. 13c).

\section{Summary and Conclusions}

A baseline load schedule for the manual calibration of a force balance was defined and evaluated. The load schedule consists of both single gage and combined loadings. The load schedule tries to best capture the physical behavior of a strain-gage balance while using equipment that is typically available for the manual calibration of a force balance.

During the evaluation of the load schedule the impact of using symmetry during the application of rolling moment loadings was demonstrated by discussing the combined load plot of the forward normal force and the rolling moment. Side force loadings at the BMC were studied to a certain degree. The determination 
of the natural zeros is also an important part of the calibration of a strain-gage balance. Therefore, three independent approaches were discussed that may be used to determine the natural zeros of a strain-gage balance. The numerical prediction of the weight of a balance from the outputs of the natural zero points was reviewed as well. This analysis may be used as an optional check of the load prediction accuracy of a data reduction matrix that is derived from a balance calibration data set.

Finally, machine calibration data was used to better understand limitations of a data reduction matrix that is derived from the proposed baseline load schedule. This evaluation showed that a matrix derived from the baseline load schedule may not perform well if a balance experiences complex combined loadings or large gage interactions during a wind tunnel test. Consequently, the authors recommend to use data from a balance calibration machine like NASA ARC's Sandberg Serell or Triumph's ABCS for the development of a data reduction matrix whenever the influence of gage interactions on the accuracy of balance load predictions needs to be minimized. This requirement becomes very important, for example, if a strain-gage balance is used for stability \& control tests.

\section{Acknowledgements}

The authors would like to thank Chris Chen and Jordan Hu of the NASA Ames Balance Calibration Laboratory and Chris Lynn of NASA Langley Research Center for providing the calibration data sets of the MK29A and MC60D balances. Thanks also go to Jon Bader of NASA Ames Research Center and Tom Volden of Jacobs Technology for their critical and constructive review of the final manuscript. The work reported in this paper was supported by the Wind Tunnel Division at NASA Ames Research Center under contract NNA09DB39C.

\section{References}

${ }^{1}$ AIAA/GTTC Internal Balance Technology Working Group, "Recommended Practice, Calibration and Use of Internal Strain-Gage Balances with Application to Wind Tunnel Testing," AIAA R-091-2003, American Institute of Aeronautics and Astronautics, Reston, Virginia, 2003.

${ }^{2}$ Ulbrich, N., "A Universal Tare Load Prediction Algorithm for Strain-Gage Balance Calibration Data Analysis," AIAA 2011-6090, paper presented at the 47th AIAA/ASME/SAE/ASEE Joint Propulsion Conference and Exhibit, San Diego, California, July/August 2011.

${ }^{3}$ Ulbrich, N., "Regression Model Optimization for the Analysis of Experimental Data," AIAA 2009-1344, paper presented at the 47th AIAA Aerospace Sciences Meeting and Exhibit, Orlando, Florida, January 2009. 


\section{Appendix: Force Components of Rolling Moment Weights}

The application of the rolling moment loadings during the manual calibration of a balance is always associated with force components that act on the forward and aft gages whenever gravity weights and a simple rolling moment arm are used to apply the loadings. Therefore, it is necessary to determine the normal or side force components at the forward and aft gages that are caused by the gravity weights at the location of the rolling moment arm on the calibration body.

The derivation of the normal and side force components leads to similar relationships as long as the correct coordinates of the gages and of the balance moment center are used. Therefore, in order to simplify the derivation, the normal and side force components $N 1$ and $S 1$ at the forward gages are replaced by the variable $F 1$. Similarly, the normal and side force components $N 2$ and $S 2$ at the aft gages are replaced by the variable $F 2$.

Relationships between the force components $F 1$ and $F 2$ at the forward and aft gages, the total force $F$ caused by the gravity weights, and the rolling moment arm coordinate $c_{0}$ can easily be derived with the help of Fig. 14. First, the total force $F$ and the total moment $M$ at the balance moment center (BMC) are replaced by two forces $F 1$ and $F 2$ at the location of the gages. The sum of these two forces equals the total force caused by the gravity weights. We get:

$$
F=F 1+F 2
$$

Similarly, we know that the total moment at the BMC equals the sum of the moment contributions from the two forces that replace the force and moment at the BMC. It is assumed that the location of both the BMC and of the gages is described in a one-dimensional coordinate system. This coordinate system is placed on the line that is defined by the roll axis of the balance (see Fig. 14). Then, the BMC has the coordinate $\mu$, the center of the rolling moment arm the coordinate $c_{0}$, the center of the forward gage the coordinate $c_{1}$, and the center of the aft gage the coordinate $c_{2}$. Now, three coordinate differences $d_{0}, d_{1}$, and $d_{2}$ may be defined that describe distances between the BMC and the forces $F, F 1$, and $F 2$. We get:

$$
\begin{aligned}
& d_{0}=\mu-c_{0} \\
& d_{1}=\mu-c_{1} \\
& d_{2}=\mu-c_{2}
\end{aligned}
$$

In the next step, using the sign definitions of (i) the balance loads and of (ii) the coordinates that are depicted in Fig. 14, the total moment at the BMC can be expressed as follows:

$$
M=F 1 \cdot d_{1}-F 2 \cdot\left(-d_{2}\right)
$$

From Eq. (1) we know that:

$$
F 2=F-F 1
$$

The right hand side of Eq. (4) may be used to replace the force $F 2$ in Eq. (3). We get:

$$
M=F 1 \cdot d_{1}-(F-F 1) \cdot\left(-d_{2}\right)
$$

Rearranging terms in Eq. (5) and after some algebra we get:

$$
F 1=F \cdot \frac{\left(-d_{2}\right)}{d_{1}-d_{2}}+M \cdot \frac{1}{d_{1}-d_{2}}
$$

Similarly, using Eq. (6) to replace force $F 1$ in Eq. (4) and after some algebra, we get:

$$
F 2=F \cdot \frac{d_{1}}{d_{1}-d_{2}}-M \cdot \frac{1}{d_{1}-d_{2}}
$$

We also know that the moment at the BMC is exclusively caused by the gravity weights that are used to apply the rolling moment loadings. Therefore, we can write: 


$$
M=F \cdot d_{0}
$$

Then, using Eq. (8) to replace the moment $M$ in (6) \& (7), we get:

$$
\begin{aligned}
& F 1=F \cdot \frac{\left(-d_{2}\right)}{d_{1}-d_{2}}+F \cdot \frac{d_{0}}{d_{1}-d_{2}} \\
& F 2=F \cdot \frac{d_{1}}{d_{1}-d_{2}}-F \cdot \frac{d_{0}}{d_{1}-d_{2}}
\end{aligned}
$$

In the next step, after simplifying Eqs. $(9 a)$ and $(9 b)$ further, we get the equations:

$$
\begin{aligned}
& F 1=F \cdot \frac{d_{0}-d_{2}}{d_{1}-d_{2}} \\
& F 2=F \cdot \frac{d_{1}-d_{0}}{d_{1}-d_{2}}
\end{aligned}
$$

Now, using Eqs. $(2 a),(2 b),(2 c)$ to replace the moment arms in Eqs. (10a) and (10b) and after some algebra, we get the relationships between the force components $F 1$ and $F 2$, the total force $F$ caused by the gravity weights, and the rolling moment arm coordinate $c_{0}$ :

$$
\begin{aligned}
& F 1=F \cdot\left[\frac{c_{2}-c_{0}}{c_{2}-c_{1}}\right] \\
& F 2=F \cdot\left[\frac{c_{0}-c_{1}}{c_{2}-c_{1}}\right]
\end{aligned}
$$

The validity of Eqs. (11a) and (11b) may be examined by looking at three limiting cases and comparing the calculated force components $F 1$ and $F 2$ with corresponding expected values.

Limiting Case 1: It is assumed that the coordinate $c_{0}$ of the center of the rolling moment arm is at the forward gage location. Then, we get the relationship:

$$
c_{0}=c_{1}
$$

Now, after using Eq. (12a) to replace $c_{0}$ in Eqs. (11a) and (11b), we get:

$$
\begin{aligned}
& F 1=F \\
& F 2=0
\end{aligned}
$$

Limiting Case 2: It is assumed that the coordinate $c_{0}$ of the center of the rolling moment arm is at the aft gage location. Then, we get the relationship:

$$
c_{0}=c_{2}
$$

Now, after using Eq. (13a) to replace $c_{0}$ in Eqs. (11a) and (11b), we get:

$$
\begin{aligned}
& F 1=0 \\
& F 2=F
\end{aligned}
$$


Limiting Case 3: It is assumed that (i) the coordinate $c_{0}$ of the center of the rolling moment arm is at the BMC of the balance and that (ii) the BMC is located halfway between the forward and aft gage. Then, we get the relationship:

$$
c_{0}=\frac{c_{1}+c_{2}}{2}
$$

Now, after using Eq. (14a) to replace $c_{0}$ in Eqs. (11a) and (11b), we get:

$$
\begin{aligned}
& F 1=\frac{F}{2} \\
& F 2=\frac{F}{2}
\end{aligned}
$$

Results for the three limiting cases, i.e., Eqs. (12b), (12c), (13b), (13c), (14b), (14c), meet expectations as (i) the total force has to equal the corresponding force at a gage location whenever the coordinate of the center of the rolling moment arm matches the coordinate of a gage and (ii) the total force is equally divided between the forces at the gage locations whenever the coordinate of the center of the rolling moment arm is halfway between the coordinates of the forward and aft gages.

The sign of $F$, i.e., of the total normal or side force caused by the gravity weights, is determined by the orientation that the balance has during the calibration (see again Fig. 1a for the definition of the orientations). Four cases have to be distinguished. Case 1 assumes that the balance orientation is 0 . In that case, $F$ has a positive sign as the gravitational acceleration $\vec{g}$ points in the direction of positive $N F$. Case 2 assumes that the balance orientation of the balance is 180 . Then, $F$ has a negative sign as the gravitational acceleration $\vec{g}$ points in the direction of negative $N F$. Case 3 assumes that the balance orientation is 90 . In that case, $F$ has a positive sign as the gravitational acceleration $\vec{g}$ points in the direction of positive $S F$. Case 4 assumes that the balance orientation of the balance is 270 . Then, $F$ has a negative sign as the gravitational acceleration $\vec{g}$ points in the direction of negative $S F$. 


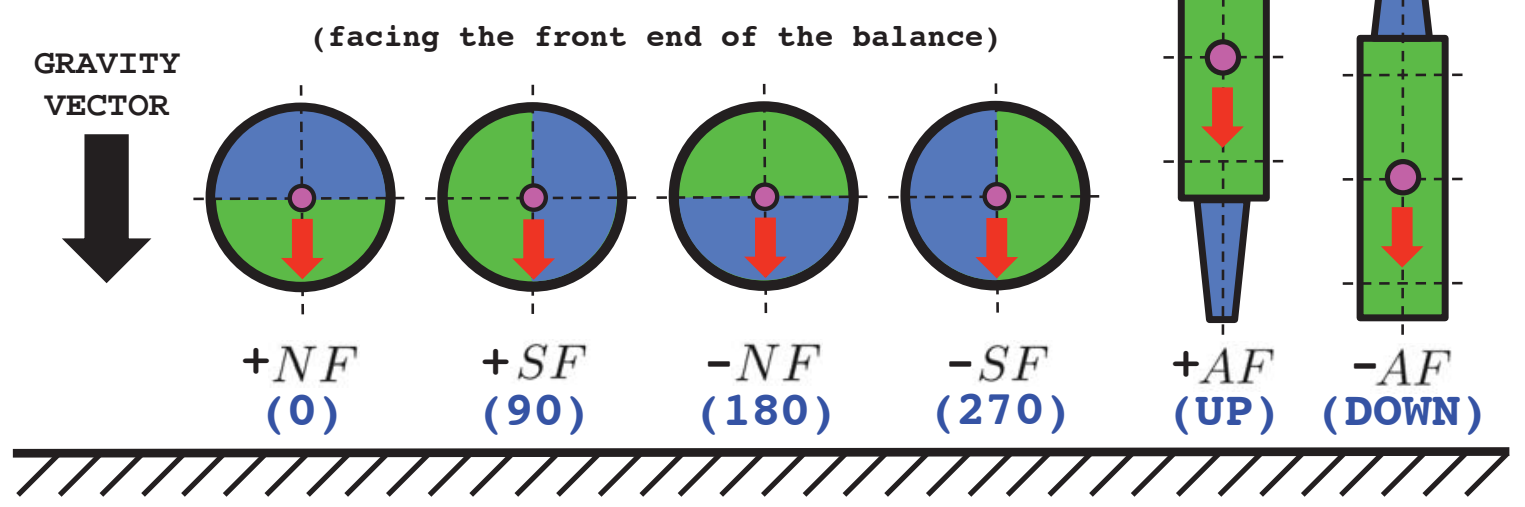

Fig. 1a Definition of balance orientations relative to the direction of the gravitational acceleration.

\begin{tabular}{|c|c|c|c|c|}
\hline Series & Orient. & Type & Position $^{\dagger}$ & List of Applied Loads in $\%$ of Capacity ${ }^{\ddagger}$ \\
\hline 1 & 0 & $+N 1 ; N 2=0$ & FWD & $0 \%,+25 \%,+50 \%,+75 \%,+100 \%,+75 \%,+50 \%,+25 \%, 0 \%$ \\
\hline 2 & 0 & $+N 2 ; N 1=0$ & AFT & $0 \%,+25 \%,+50 \%,+75 \%,+100 \%,+75 \%,+50 \%,+25 \%, 0 \%$ \\
\hline 3 & 0 & $+N 1 ;+N 2$ & $\mathrm{BMC}$ & $0 \%,+25 \%,+50 \%,+75 \%,+100 \%,+75 \%,+50 \%,+25 \%, 0 \%$ \\
\hline 4 & 180 & $-N 1 ; N 2=0$ & FWD & $0 \%,-25 \%,-50 \%,-75 \%,-100 \%,-75 \%,-50 \%,-25 \%, 0 \%$ \\
\hline 5 & 180 & $-N 2 ; N 1=0$ & AFT & $0 \%,-25 \%,-50 \%,-75 \%,-100 \%,-75 \%,-50 \%,-25 \%, 0 \%$ \\
\hline 6 & 180 & $-N 1 ;-N 2$ & $\mathrm{BMC}$ & $0 \%,-25 \%,-50 \%,-75 \%,-100 \%,-75 \%,-50 \%,-25 \%, 0 \%$ \\
\hline 7 & 90 & $+S 1 ; S 2=0$ & FWD & $0 \%,+25 \%,+50 \%,+75 \%,+100 \%,+75 \%,+50 \%,+25 \%, 0 \%$ \\
\hline 8 & 90 & $+S 2 ; S 1=0$ & AFT & $0 \%,+25 \%,+50 \%,+75 \%,+100 \%,+75 \%,+50 \%,+25 \%, 0 \%$ \\
\hline 9 & 90 & $+S 1 ;+S 2$ & $\mathrm{BMC}$ & $0 \%,+25 \%,+50 \%,+75 \%,+100 \%,+75 \%,+50 \%,+25 \%, 0 \%$ \\
\hline 10 & 270 & $-S 1 ; S 2=0$ & FWD & $0 \%,-25 \%,-50 \%,-75 \%,-100 \%,-75 \%,-50 \%,-25 \%, 0 \%$ \\
\hline 11 & 270 & $-S 2 ; S 1=0$ & AFT & $0 \%,-25 \%,-50 \%,-75 \%,-100 \%,-75 \%,-50 \%,-25 \%, 0 \%$ \\
\hline 12 & 270 & $-S 1 ;-S 2$ & $\mathrm{BMC}$ & $0 \%,-25 \%,-50 \%,-75 \%,-100 \%,-75 \%,-50 \%,-25 \%, 0 \%$ \\
\hline 13 & 0 & $\begin{array}{c} \pm R M \\
(+N F=\text { const. })\end{array}$ & $\begin{array}{c}c_{0} \\
\text { (see Fig. 14) }\end{array}$ & $\begin{array}{l}0 \%,+25 \%,+50 \%,+75 \%,+100 \%,+75 \%,+50 \%,+25 \%, 0 \% \text {, } \\
-25 \%,-50 \%,-75 \%,-100 \%,-75 \%,-50 \%,-25 \%, 0 \%\end{array}$ \\
\hline 14 & 180 & $\begin{array}{c} \pm R M \\
(-N F=\text { const. })\end{array}$ & $\begin{array}{c}c_{0} \\
\text { (see Fig. 14) }\end{array}$ & $\begin{array}{l}0 \%,+25 \%,+50 \%,+75 \%,+100 \%,+75 \%,+50 \%,+25 \%, 0 \% \text {, } \\
-25 \%,-50 \%,-75 \%,-100 \%,-75 \%,-50 \%,-25 \%, 0 \%\end{array}$ \\
\hline 15 & 90 & $\begin{array}{c} \pm R M \\
(+S F=\text { const. })\end{array}$ & $\begin{array}{c}c_{0} \\
\text { (see Fig. 14) }\end{array}$ & $\begin{array}{l}0 \%,+25 \%,+50 \%,+75 \%,+100 \%,+75 \%,+50 \%,+25 \%, 0 \%, \\
-25 \%,-50 \%,-75 \%,-100 \%,-75 \%,-50 \%,-25 \%, 0 \%\end{array}$ \\
\hline 16 & 270 & $\begin{array}{c} \pm R M \\
(-S F=\text { const. })\end{array}$ & $\begin{array}{c}c_{0} \\
\text { (see Fig. 14) }\end{array}$ & $\begin{array}{l}0 \%,+25 \%,+50 \%,+75 \%,+100 \%,+75 \%,+50 \%,+25 \%, 0 \% \text {, } \\
-25 \%,-50 \%,-75 \%,-100 \%,-75 \%,-50 \%,-25 \%, 0 \%\end{array}$ \\
\hline 17 & UP & $+A F$ & - & $0 \%,+25 \%,+50 \%,+75 \%,+100 \%,+75 \%,+50 \%,+25 \%, 0 \%$ \\
\hline 18 & DOWN & $-A F$ & - & $0 \%,-25 \%,-50 \%,-75 \%,-100 \%,-75 \%,-50 \%,-25 \%, 0 \%$ \\
\hline
\end{tabular}

${ }^{\dagger} \mathrm{FWD}=$ load acts at the $\underline{\text { forward gage }} ; \mathrm{AFT}=$ load acts at the aft gage $; \mathrm{BMC}=$ load acts at the balance moment center.

${ }_{\ddagger}^{\ddagger}$ The spacing of $25 \%$ is used to indicate that at least three loads should be applied between $0 \%$ and $100 \%$ of capacity; a load of $\pm 100 \%$ of capacity at the $\underline{\mathrm{BMC}}$ means that loads at the forward and aft gages are simultaneously at $\pm 100 \%$ of capacity.

Fig. 1b Baseline load schedule for the manual calibration of a six-component force balance. 


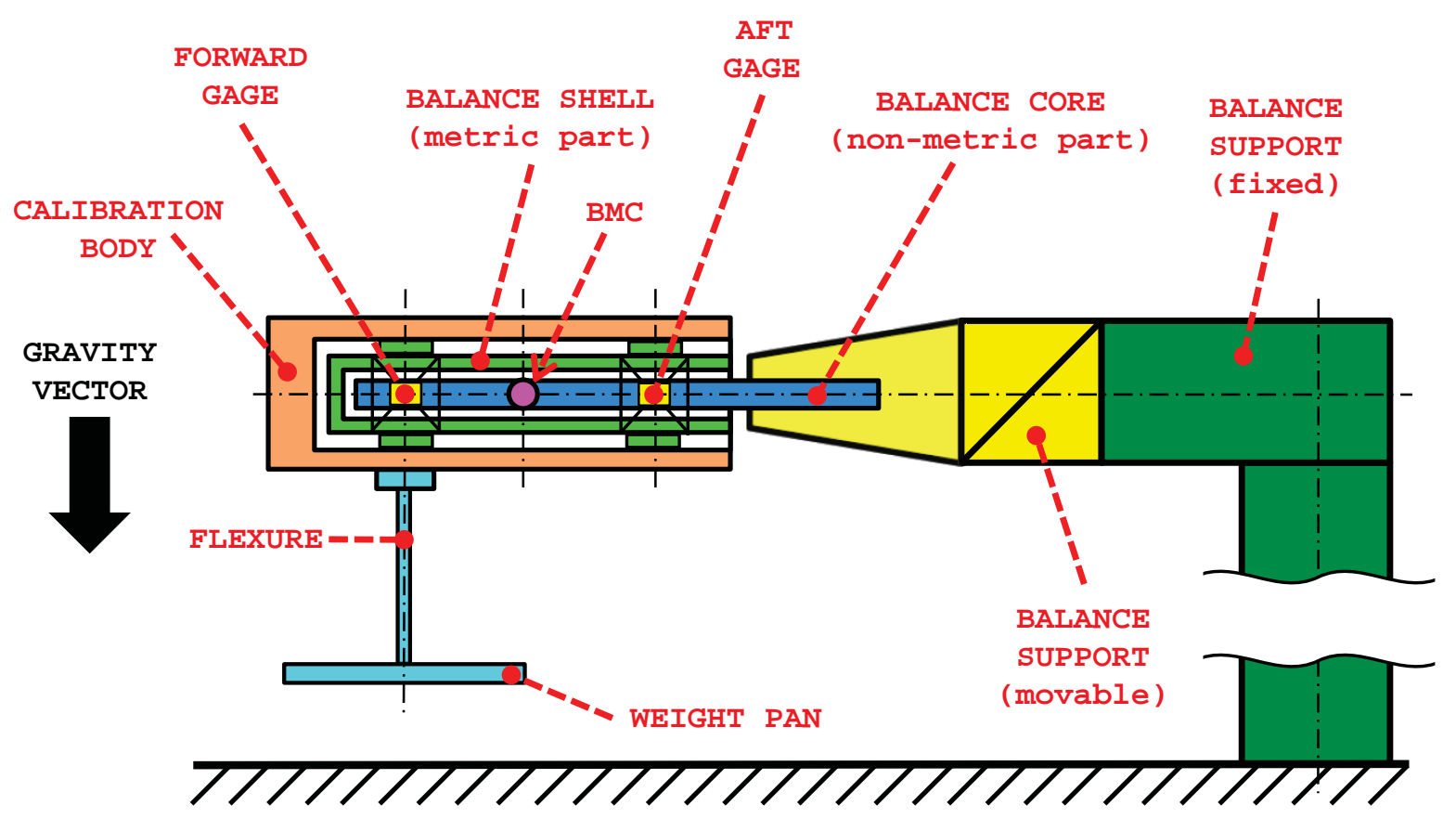

Fig. 2a Setup of balance, calibration hardware, and support for normal or side force loadings.

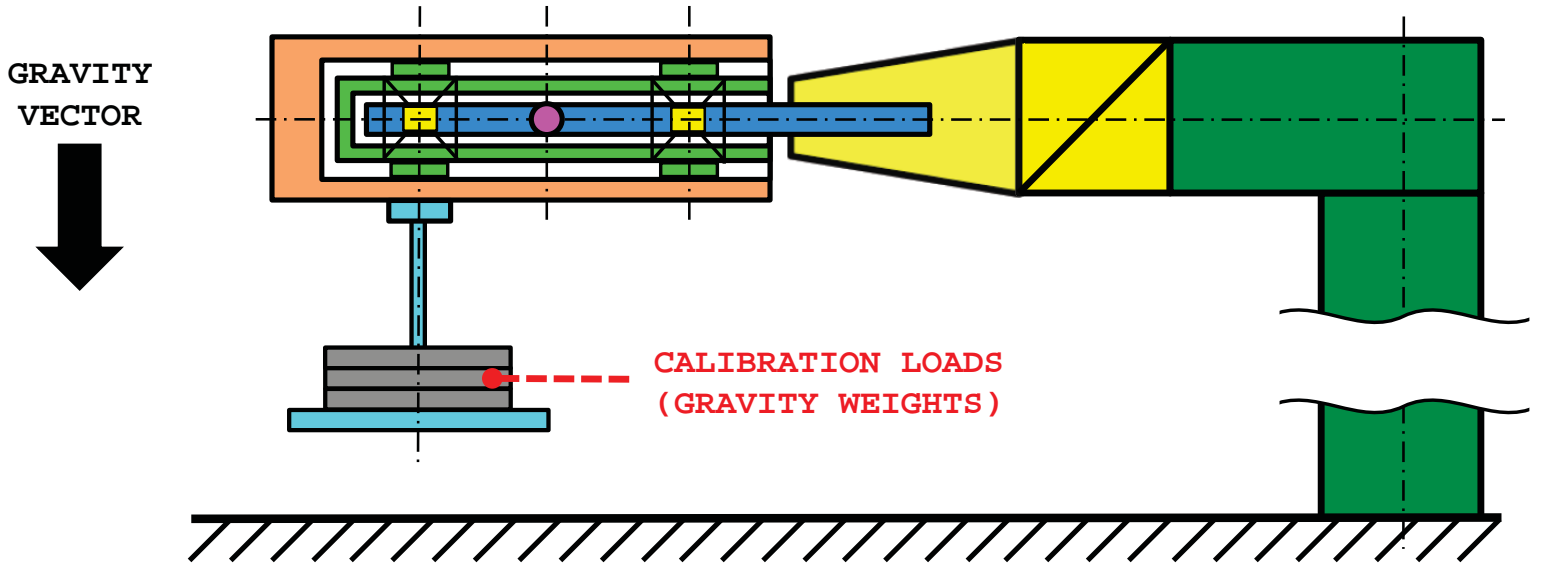

Fig. 2b Application of normal or side force loadings using gravity weights. 


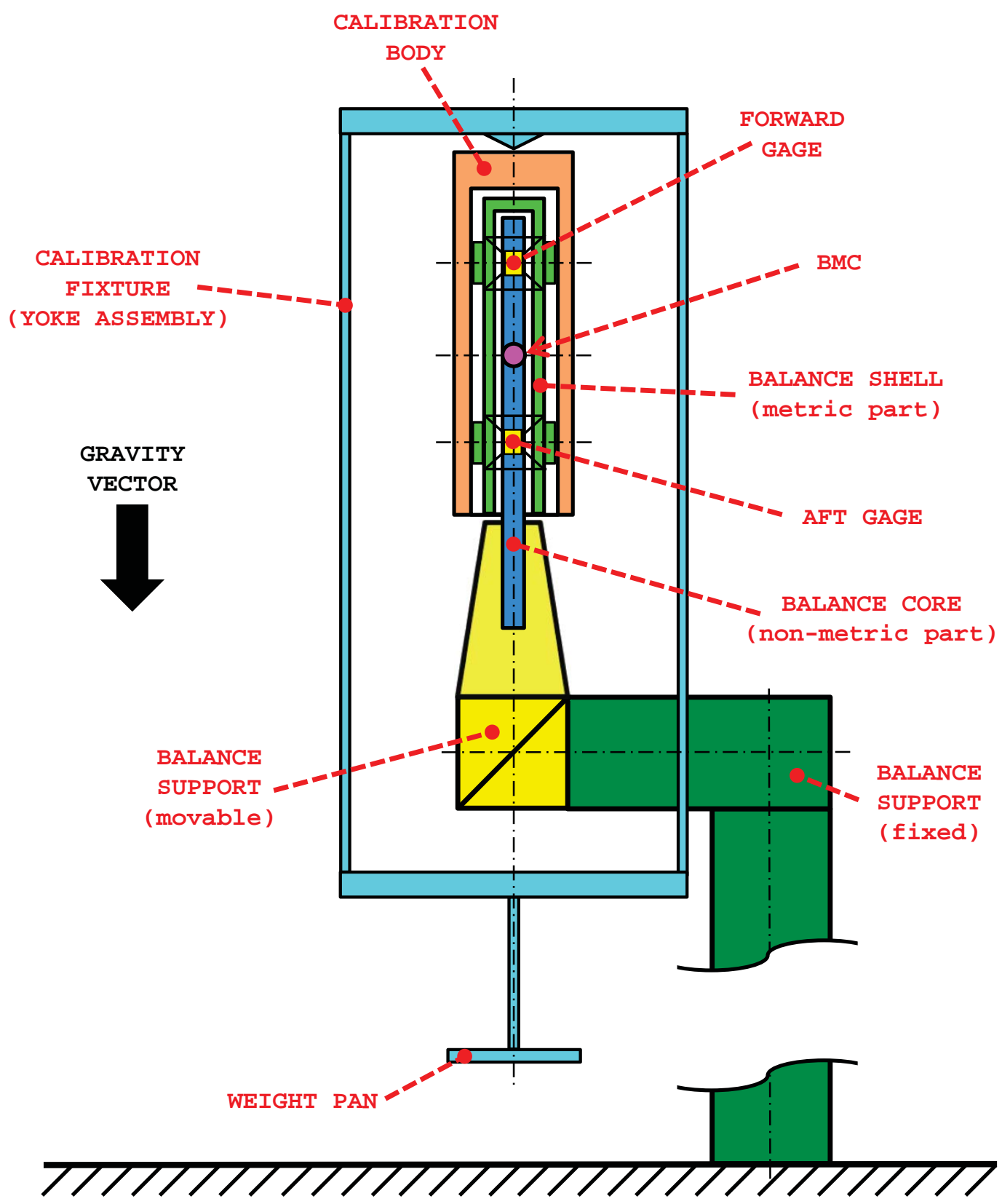

Fig. 3a Setup of balance, calibration hardware, and support for positive axial force loadings. 


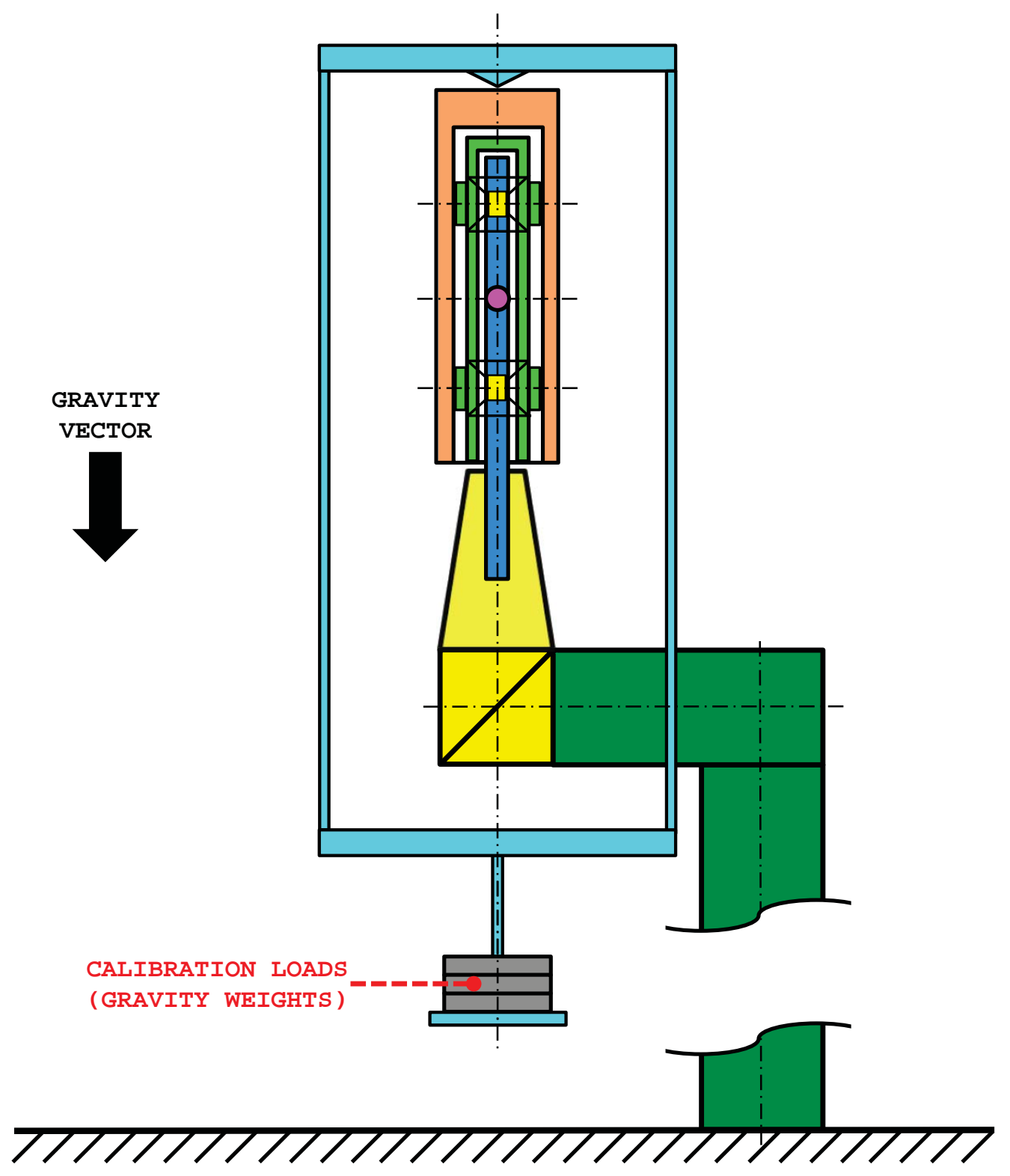

Fig. 3b Application of positive axial force loadings using gravity weights. 


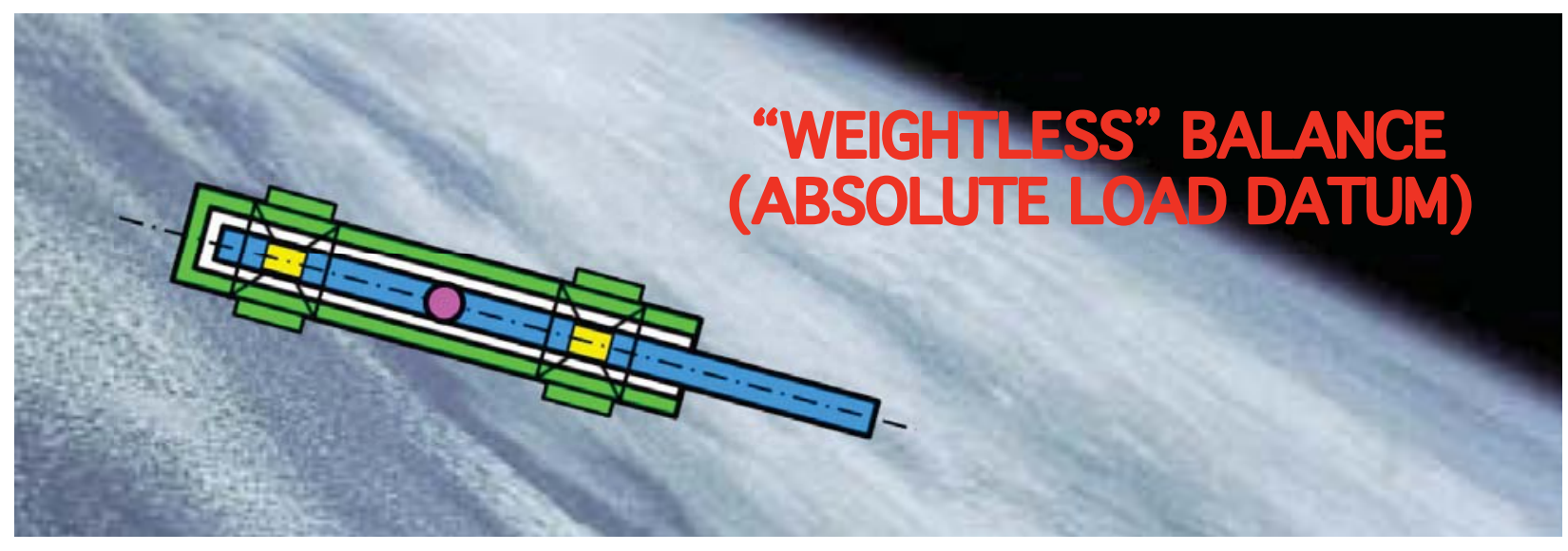

Fig. 4a Definition of the absolute load datum of a strain-gage balance.

\begin{tabular}{|c|c|c|c|}
\hline Method & Orientations & Acting Balance Load & Mounted/Supported Balance Assembly \\
\hline A & $\begin{array}{c}0-\mathrm{S}, 90-\mathrm{S} \\
180-\mathrm{S}, 270-\mathrm{S}\end{array}$ & $\begin{array}{c}\text { weight of shell (metric part), or, weight of } \\
\text { shell assembly (calibration body and metric part) }\end{array}$ & $\frac{\text { core }}{(\text { non-metric part) }}$ \\
\hline B & $\begin{array}{c}\text { UP-S, } \\
\text { DOWN-S }\end{array}$ & $\begin{array}{l}\text { weight of shell (metric part), or, weight of } \\
\text { shell assembly (calibration body and metric part) }\end{array}$ & $\frac{\text { core }}{\text { (non-metric part) }}$ \\
\hline $\mathrm{C}$ & $\begin{array}{c}0-\mathrm{C}, 90-\mathrm{C} \\
180-\mathrm{C}, 270-\mathrm{C}\end{array}$ & $\begin{array}{l}\text { weight of core } \\
\text { (non-metric part) }\end{array}$ & $\frac{\text { shell (metric part), or, shell assembly }}{\text { (calibration body and metric part) }}$ \\
\hline
\end{tabular}

Fig. 4b Comparison of three methods for the determination of the natural zeros.

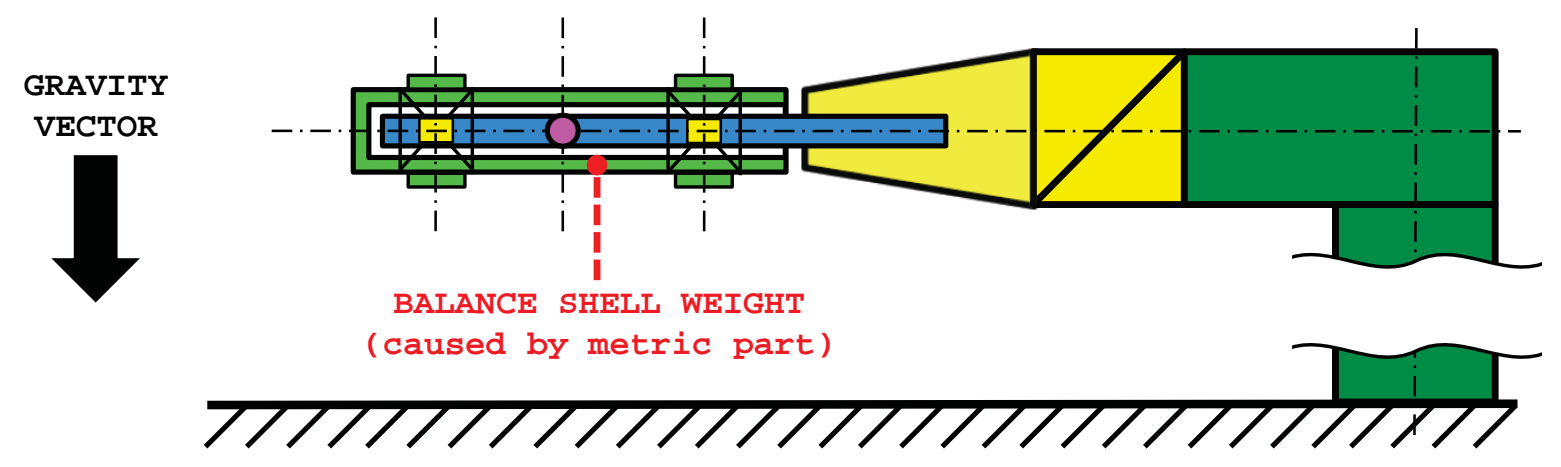

Fig. 5a Method A: Setup of balance without use of calibration body for horizontal alignment. 


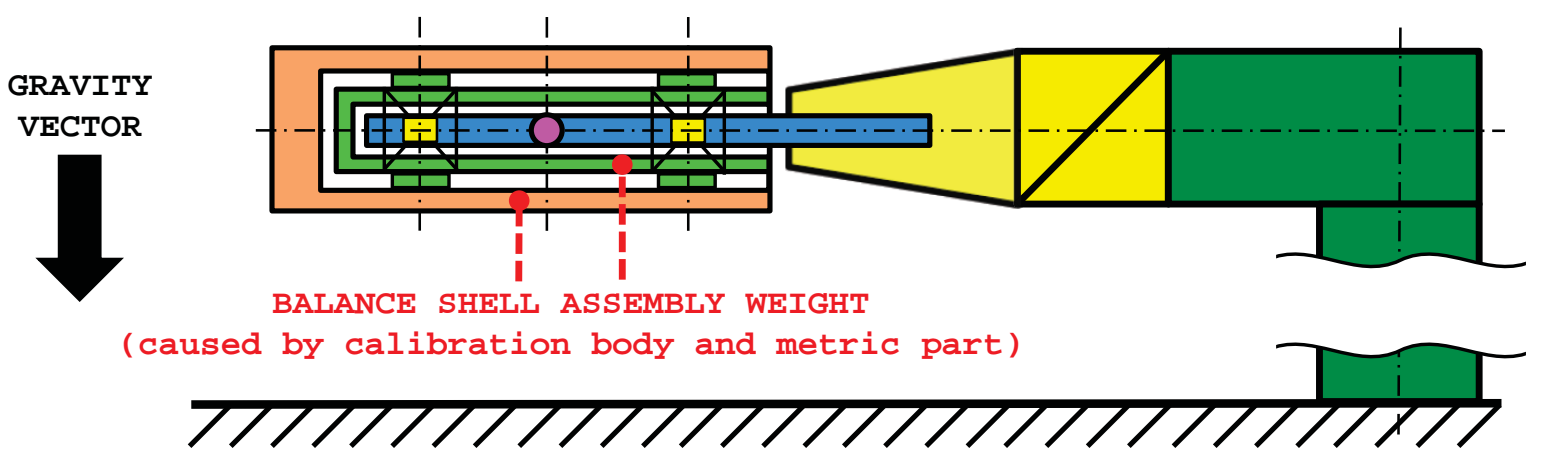

Fig. 5b Method A: Setup of balance with use of calibration body for horizontal alignment.

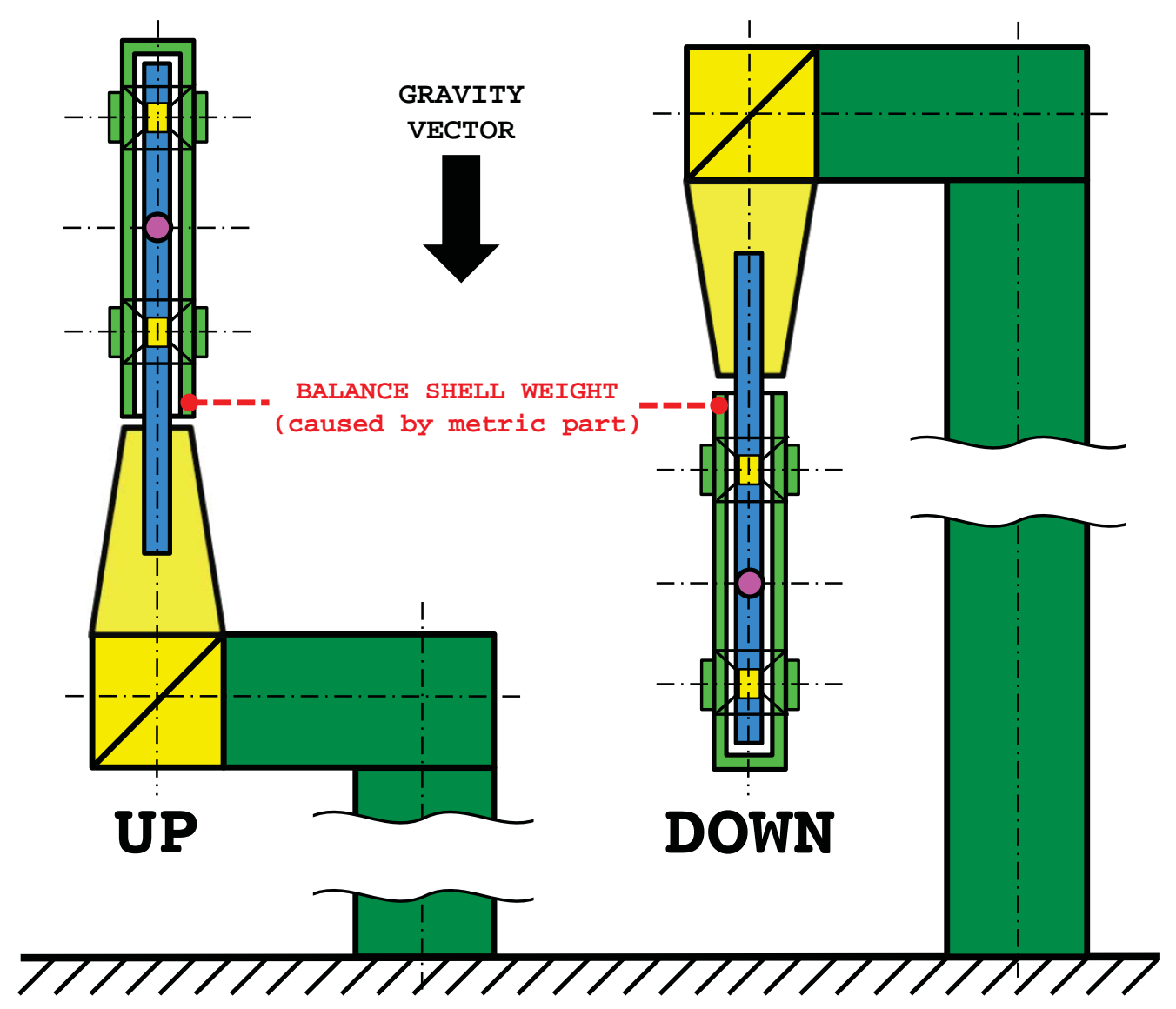

Fig. 6a Method B: Setup of balance without use of calibration body for vertical alignment. 


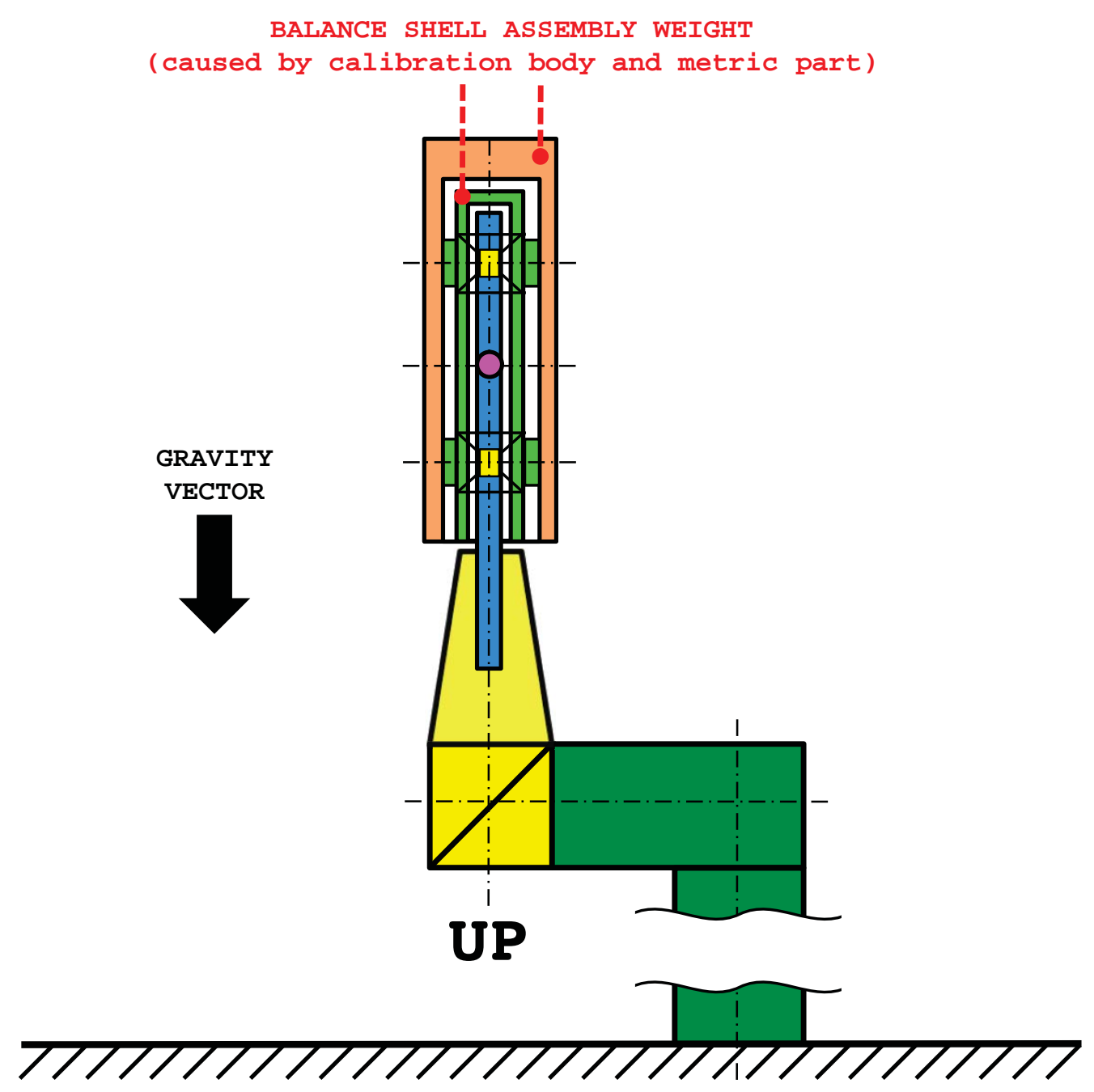

Fig. 6b Method B: Setup of balance with use of calibration body for vertical alignment. 


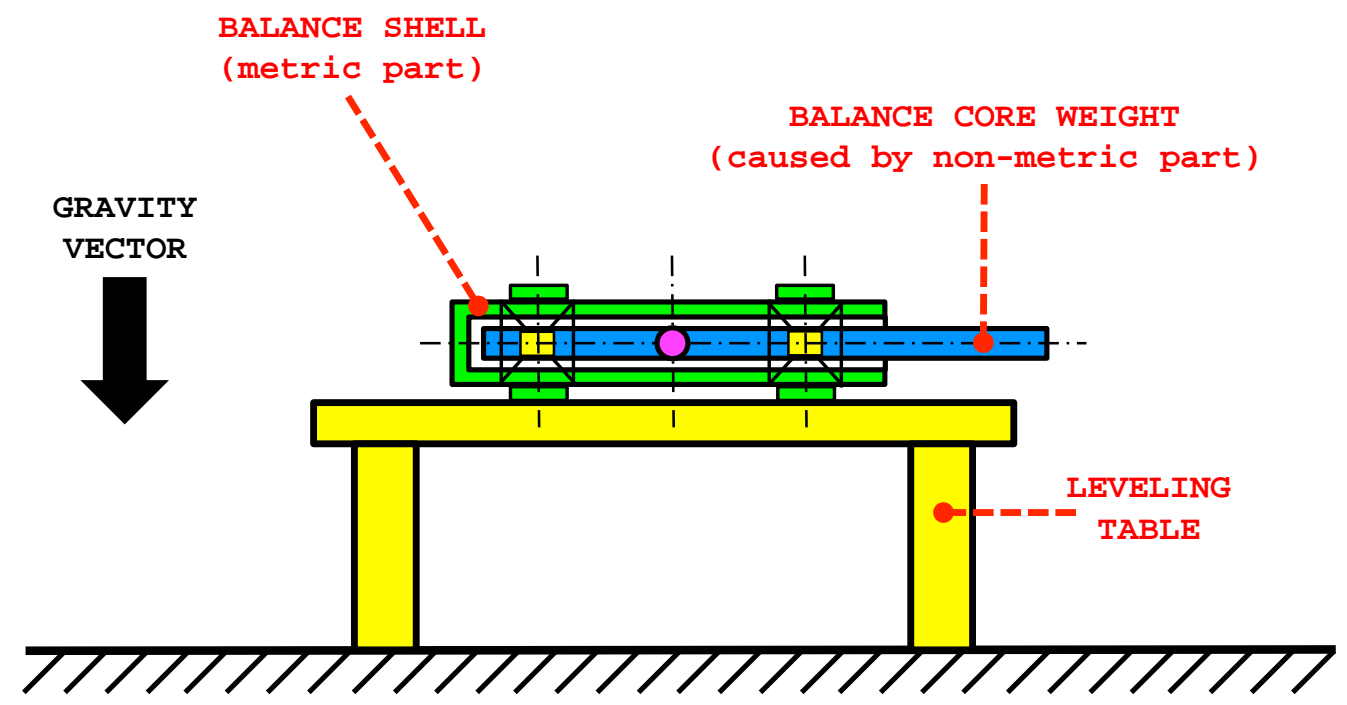

Fig. 7a Method C: Setup of balance on a leveling table (vee block not shown).

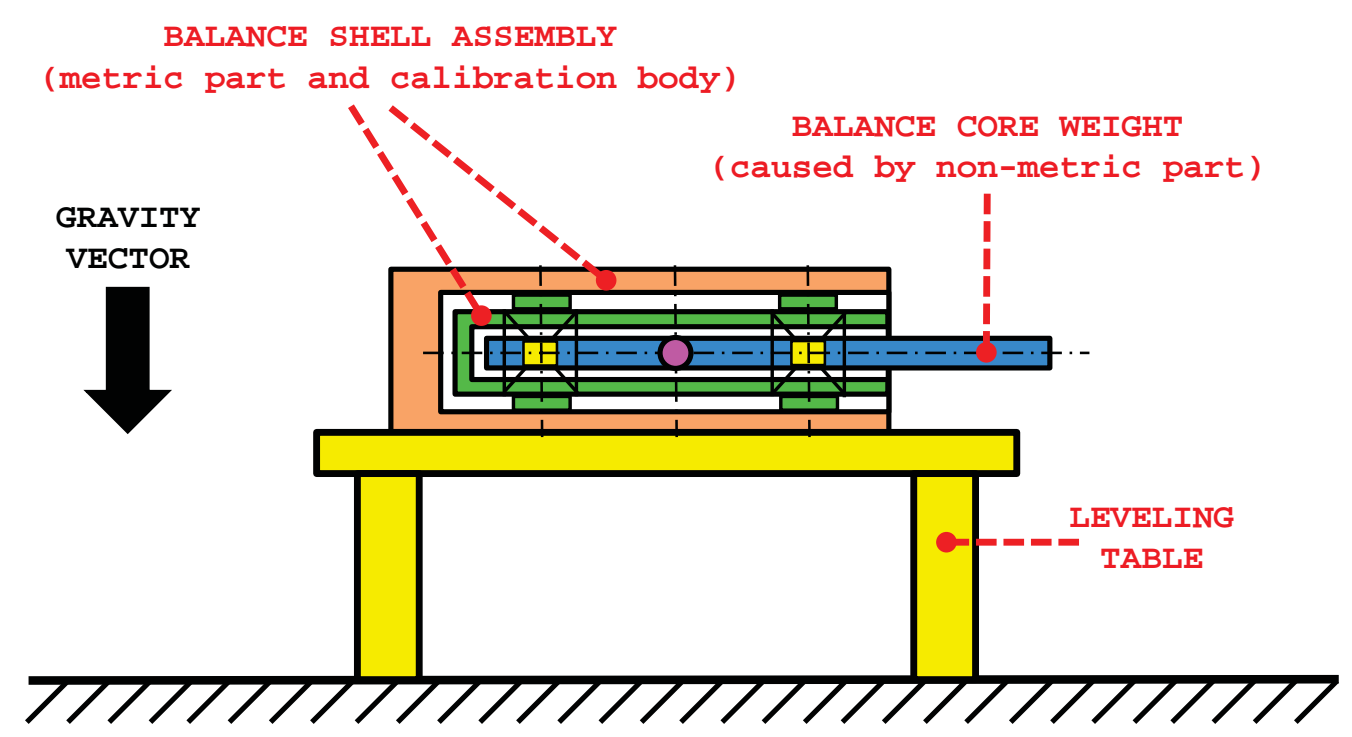

Fig. 7b Method C: Setup of balance and calibration body on a leveling table. 

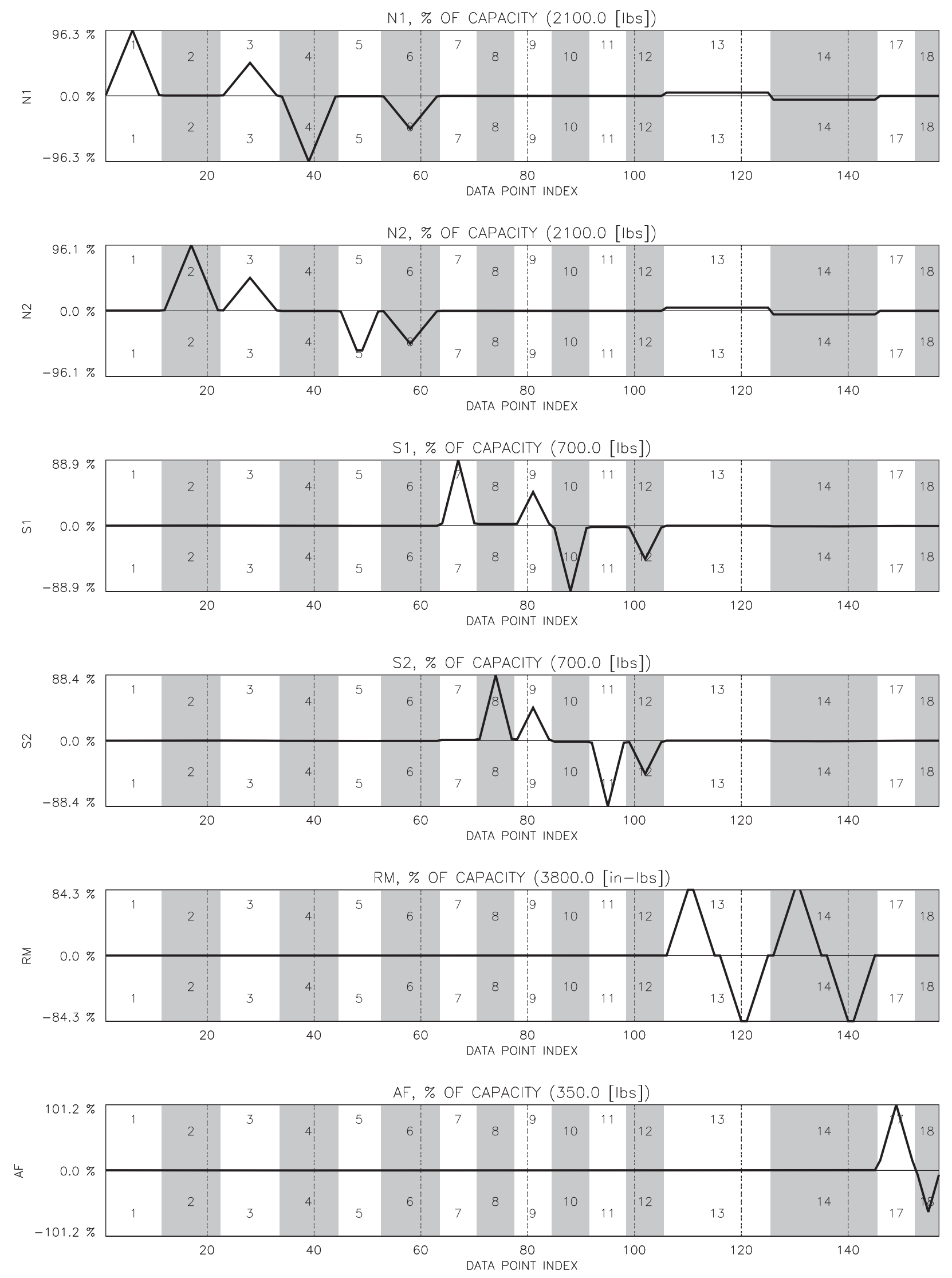

Fig. 8 MK29A: Load schedule of the 157-point manual calibration of the balance. 


\section{TABLE OF GAGE OUTPUTS OF NATURAL ZERO POINTS}

\begin{tabular}{|c|c|c|c|c|c|c|c|c|}
\hline \multicolumn{9}{|c|}{ (listed gage outputs were read from data input file) } \\
\hline INDEX & IDENT. & ORIENT. & R1 & R2 & R3 & R4 & R5 & R6 \\
\hline--- & --- & --- & microv/V & microv/V & microv/V & microv/V & microv/V & microv/V \\
\hline 1 & $N Z-0001$ & $0-S$ & +42.7073 & -41.3250 & -24.9820 & -56.9415 & +37.3324 & -564.6451 \\
\hline 2 & $N Z-0002$ & $90-S$ & +43.4122 & -40.6998 & -27.0981 & -58.7094 & +37.5553 & -564.5992 \\
\hline 3 & $N Z-0003$ & $180-5$ & +44.0595 & -40.2123 & -24.8009 & -56.8479 & +37.5037 & -564.7283 \\
\hline 4 & $N Z-0004$ & $270-5$ & +43.3519 & -40.7634 & -22.5458 & -55.0322 & +37.2246 & -564.7114 \\
\hline 5 & $N Z-0005$ & UP-S & +43.3703 & -40.6200 & -25.0041 & -56.8452 & +37.3925 & -552.8320 \\
\hline 6 & $N Z-0006$ & DOWN-S & +43.5367 & -40.7239 & -24.8094 & -56.8439 & +37.3644 & -575.9301 \\
\hline 7 & $N Z-0007$ & $0-C$ & +44.1058 & -39.0955 & -24.9523 & -56.7286 & +37.4433 & -564.9438 \\
\hline 8 & $N Z-0008$ & $90-C$ & +43.5221 & -40.4844 & -23.4147 & -51.9700 & +37.5735 & -564.9536 \\
\hline 9 & $N Z-0009$ & $180-C$ & +42.9868 & -42.0328 & -25.1137 & -56.7785 & +37.4167 & -564.7876 \\
\hline 10 & $N Z-0010$ & $270-C$ & +43.4731 & -40.8346 & -26.3740 & -60.8948 & +37.2094 & -564.8133 \\
\hline
\end{tabular}

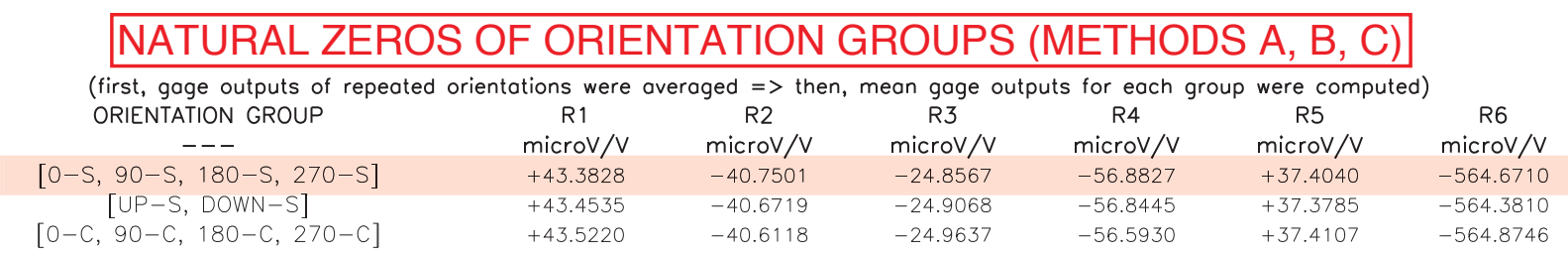

\section{GLOBAL NATURAL ZEROS (MEAN OF METHODS A, B, C)}

\begin{tabular}{|c|c|c|c|c|c|}
\hline \multicolumn{6}{|c|}{ (computed as the arithmetic mean of the natural zeros of all orientation groups) } \\
\hline $\begin{array}{c}\mathrm{R} 1 \\
\mathrm{microV} / \mathrm{V}\end{array}$ & $\begin{array}{c}\mathrm{R} 2 \\
\mathrm{microV} / \mathrm{V}\end{array}$ & $\begin{array}{c}\mathrm{R3} \\
\mathrm{microV} / \mathrm{V}\end{array}$ & $\begin{array}{c}\mathrm{R} 4 \\
\mathrm{microV} / \mathrm{V}\end{array}$ & $\begin{array}{c}\text { R5 } \\
\text { microV/V }\end{array}$ & $\begin{array}{c}\mathrm{R} 6 \\
\mathrm{microV} / \mathrm{V}\end{array}$ \\
\hline+43.4527 & -40.6780 & -24.9091 & -56.7734 & +37.3977 & -564.6422 \\
\hline
\end{tabular}

Fig. 9a MK29A: Natural zero points for Method A, Method B, and Method C. ( $\underline{\text { red }}$ region $\Longrightarrow \underline{\text { Method } A}$; green region $\Longrightarrow$ final natural zeros)

\begin{tabular}{|c|c|c|c|c|c|c|c|c|}
\hline \multicolumn{9}{|c|}{$\begin{array}{l}\text { GAGE OUTPUT DIFFERENCES CAUSED BY BALANCE SHELL OR CORE } \\
\text { (defined as differences between the gage outputs caused by the shell, shell assembly, or core and the natural zeros) }\end{array}$} \\
\hline INDEX & IDENT. & ORIENT. & R1 & R2 & R3 & R4 & R5 & R6 \\
\hline--- & --- & --- & microv/V & microv/V & microv/V & microv/V & microv/V & microV/V \\
\hline 1 & $N Z-0001$ & $0-s$ & -0.7454 & -0.6470 & -0.0729 & -0.1681 & -0.0653 & -0.0029 \\
\hline 2 & $N Z-0002$ & $90-5$ & -0.0405 & -0.0219 & -2.1890 & -1.9359 & +0.1576 & +0.0430 \\
\hline 3 & $N Z-0003$ & $180-S$ & +0.6068 & +0.4657 & +0.1081 & -0.0745 & +0.1060 & -0.0861 \\
\hline 4 & $N Z-0004$ & $270-S$ & -0.1008 & -0.0855 & +2.3633 & +1.7412 & -0.1732 & -0.0692 \\
\hline 5 & $N Z-0005$ & UP-S & -0.0824 & +0.0580 & -0.0951 & -0.0718 & -0.0053 & +11.8102 \\
\hline 6 & $N Z-0006$ & DOWN-S & +0.0840 & -0.0460 & +0.0997 & -0.0704 & -0.0333 & -11.2879 \\
\hline 7 & $N Z-0007$ & $0-C$ & +0.6530 & +1.5825 & -0.0433 & +0.0448 & +0.0455 & -0.3016 \\
\hline 8 & $N Z-0008$ & $90-C$ & +0.0694 & +0.1935 & +1.4943 & +4.8034 & +0.1758 & -0.3114 \\
\hline 9 & $N Z-0009$ & $180-C$ & -0.4659 & -1.3549 & -0.2046 & -0.0051 & +0.0190 & -0.1454 \\
\hline 10 & $N Z-0010$ & $270-C$ & +0.0204 & -0.1566 & -1.4650 & -4.1214 & -0.1883 & -0.1711 \\
\hline
\end{tabular}

COMPONENT WEIGHTS CAUSED BY BALANCE SHELL OR CORE

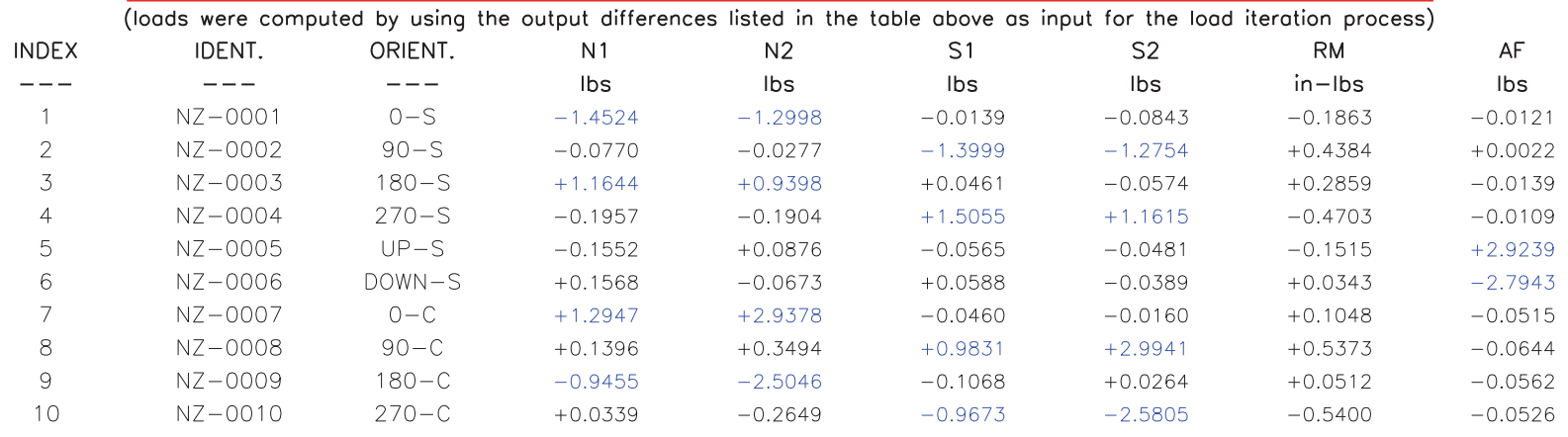

Fig. 9b MK29A: Theoretically computed balance shell (metric part) and balance core (non-metric part) weights. (blue color $\Longrightarrow$ force components that are related to balance shell or core weights)

American Institute of Aeronautics and Astronautics 


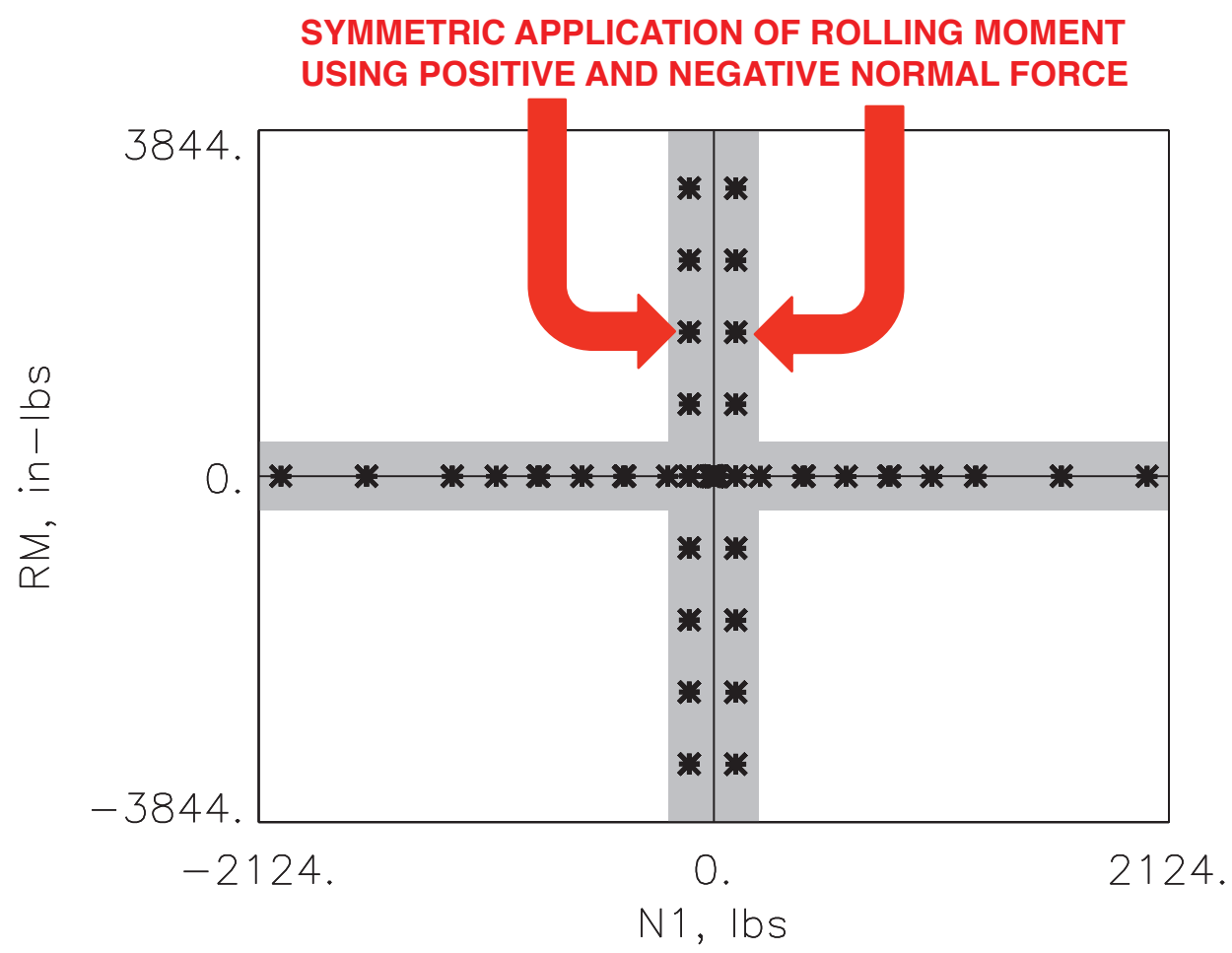

Fig. 10 MK29A: Example of "symmetry" of rolling moment load schedule.

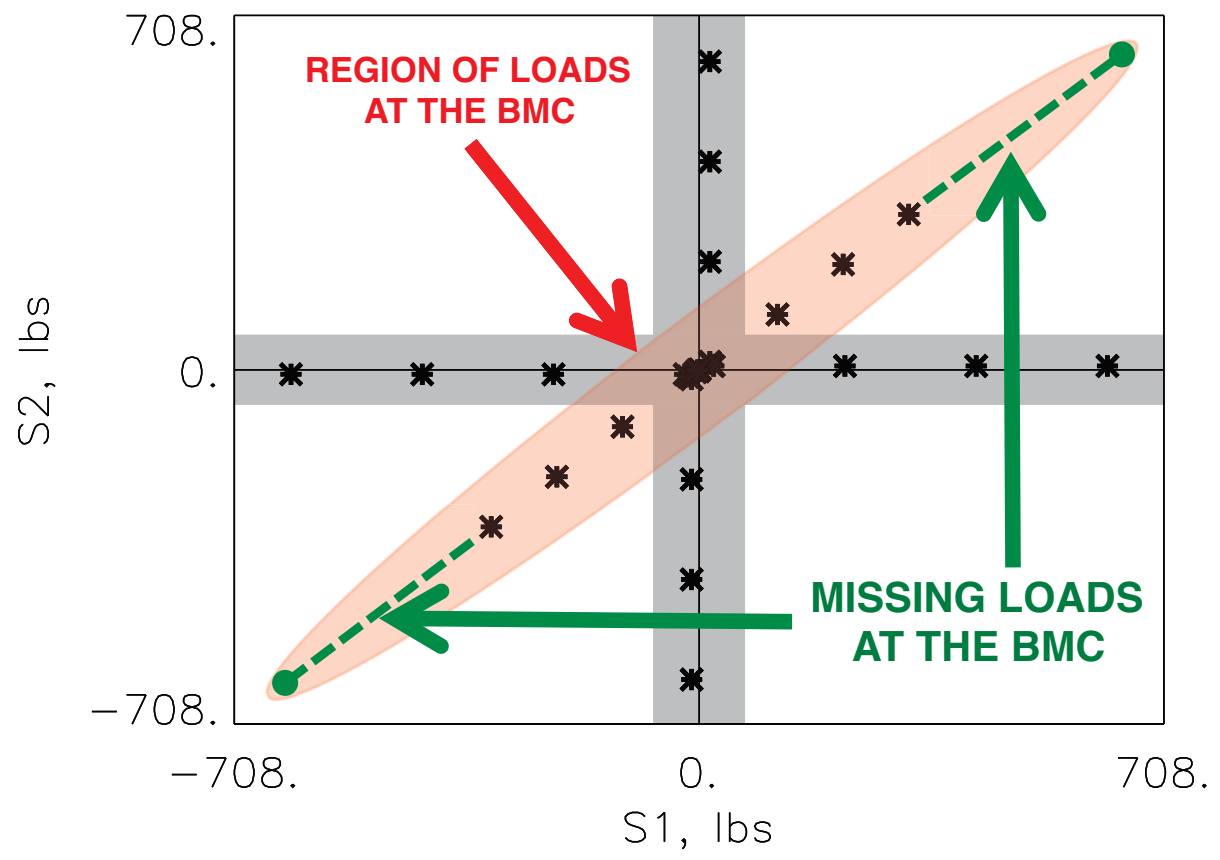

Fig. 11 MK29A: Application of side force components at the balance moment center (BMC). 
N1,\% OF CAPACITY (2500.0 [lbs])

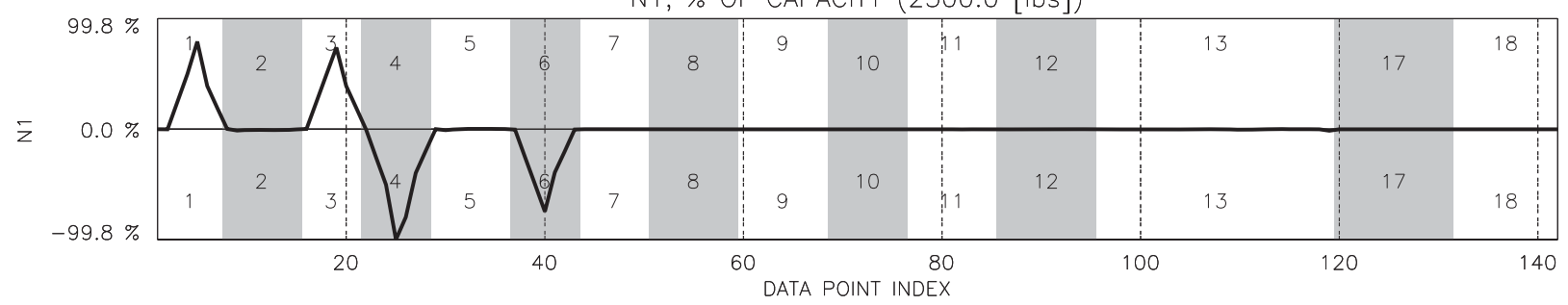

N2, \% OF CAPACITY (2500.0 [lbs])
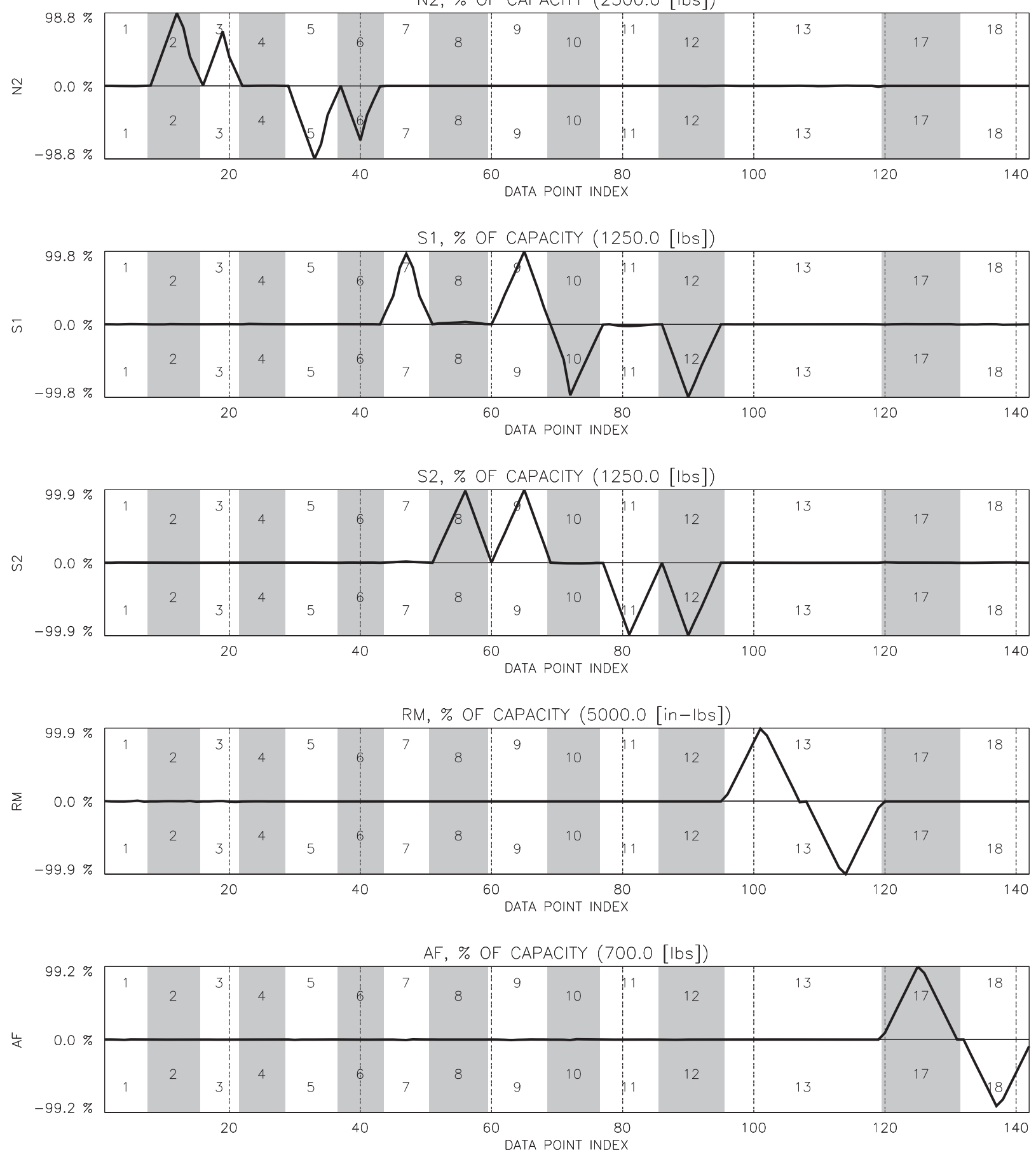

Fig. 12 MC60D: 142-point subset of original 1906-point machine calibration data set. $(142-$ point subset of machine calibration $\equiv$ assumed manual calibration data) 
$\Delta \mathrm{N} 1$

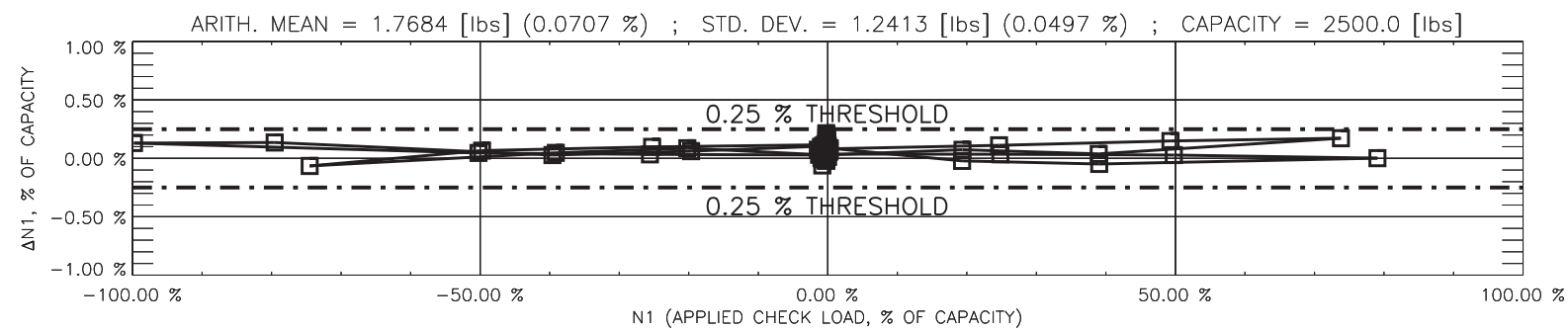

$\Delta \mathrm{N} 2$

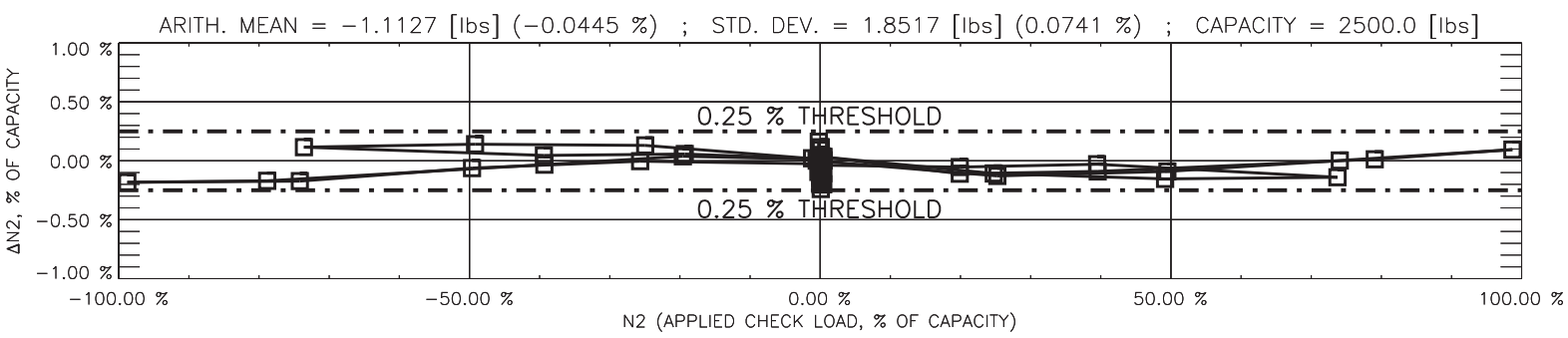

$\Delta \mathrm{S} 1$

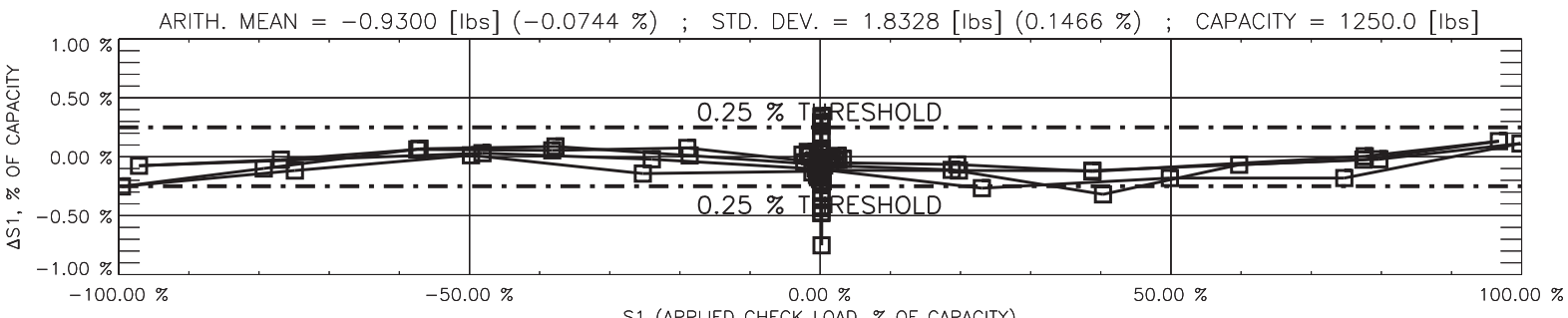

$\Delta \mathrm{S} 2$

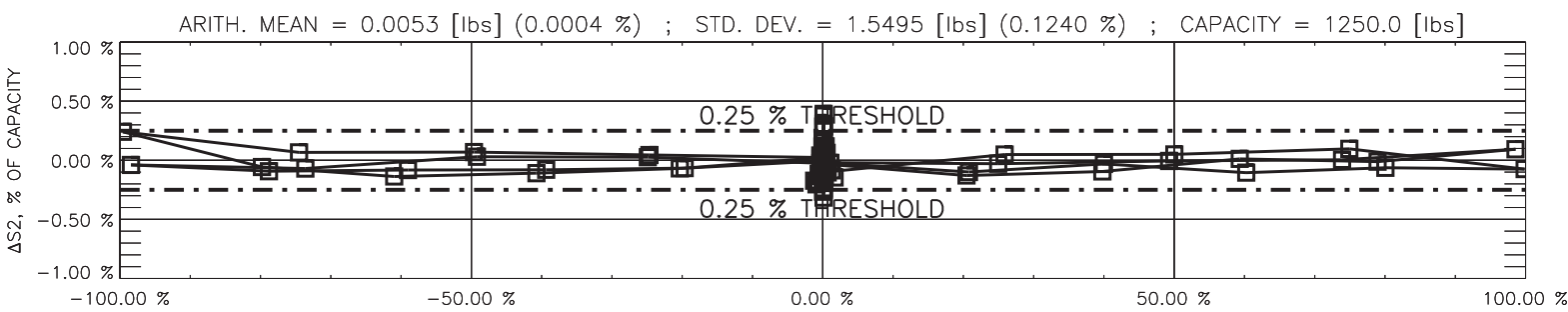

S2 (APPLIED CHECK LOAD, \% OF CAPACITY)

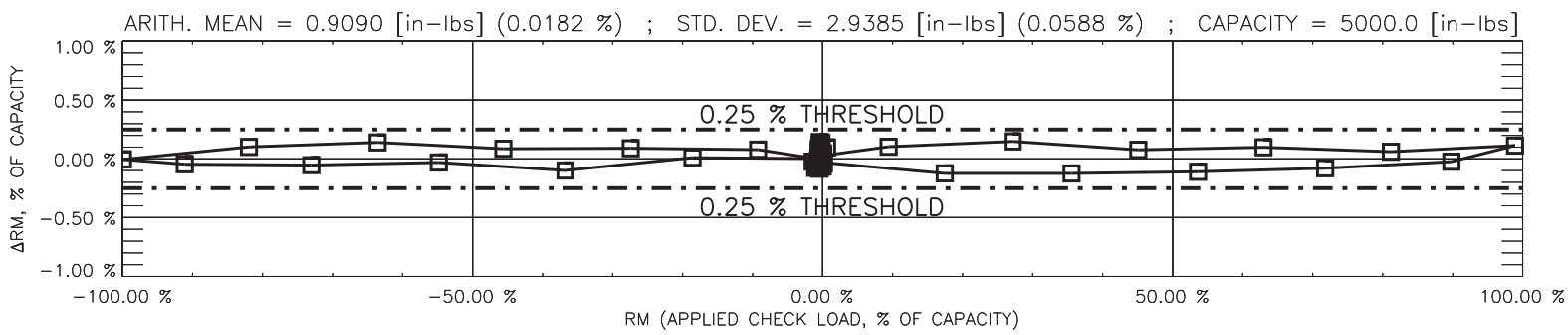

$\Delta \mathrm{RM}$

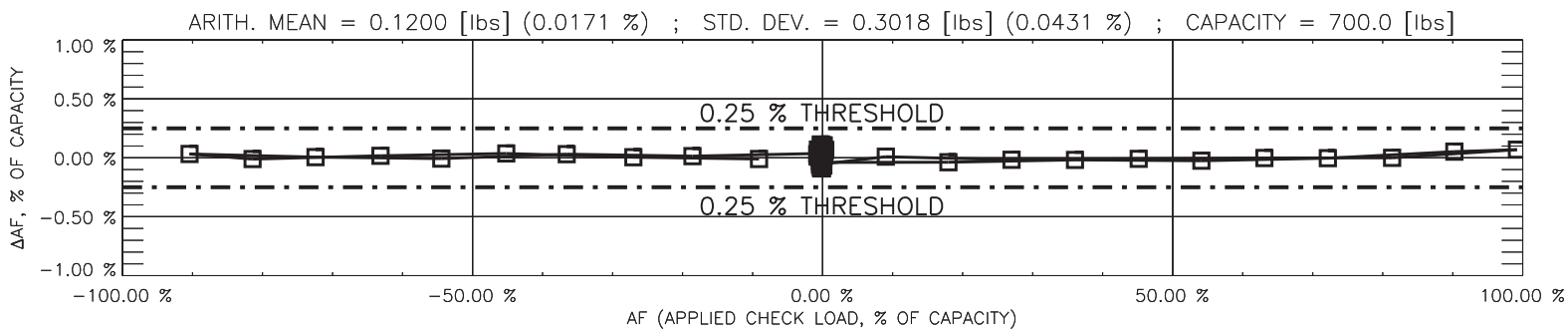

Fig. 13a MC60D: Load residuals of 142-point subset after application of matrix of 142-point subset (matrix 1).

American Institute of Aeronautics and Astronautics 
$\Delta \mathrm{N} 1$

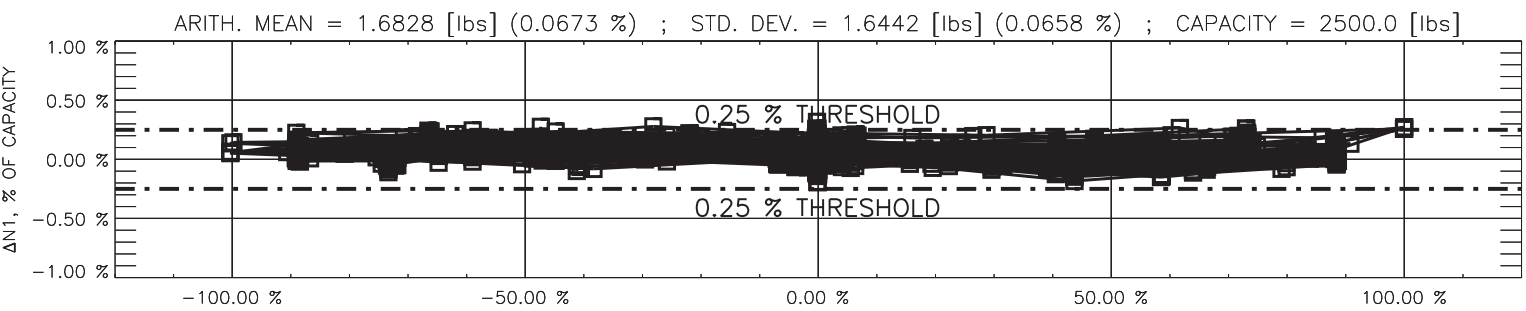

N1 (APPLIED CHECK LOAD, \% OF CAPACITY)

$\Delta \mathrm{N} 2$

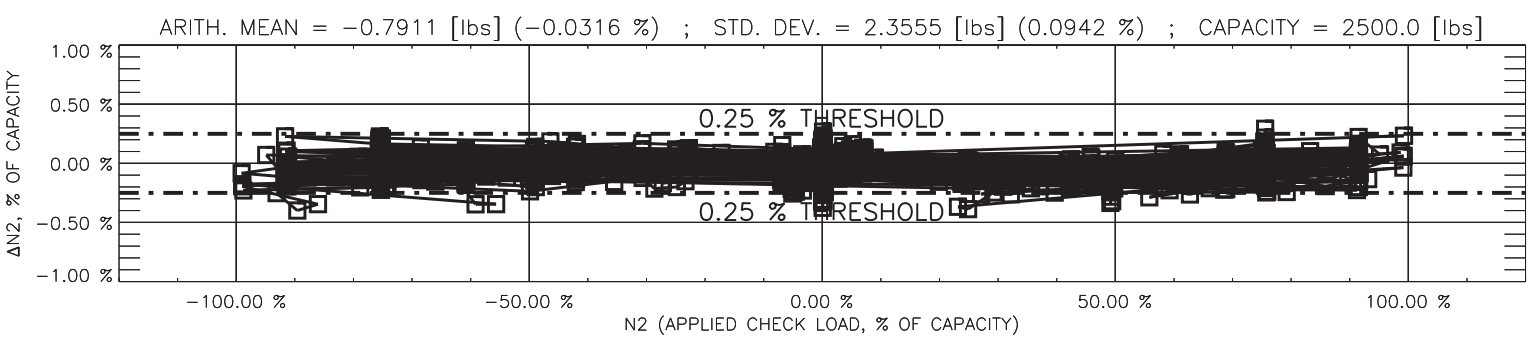

$\Delta \mathrm{S} 1$

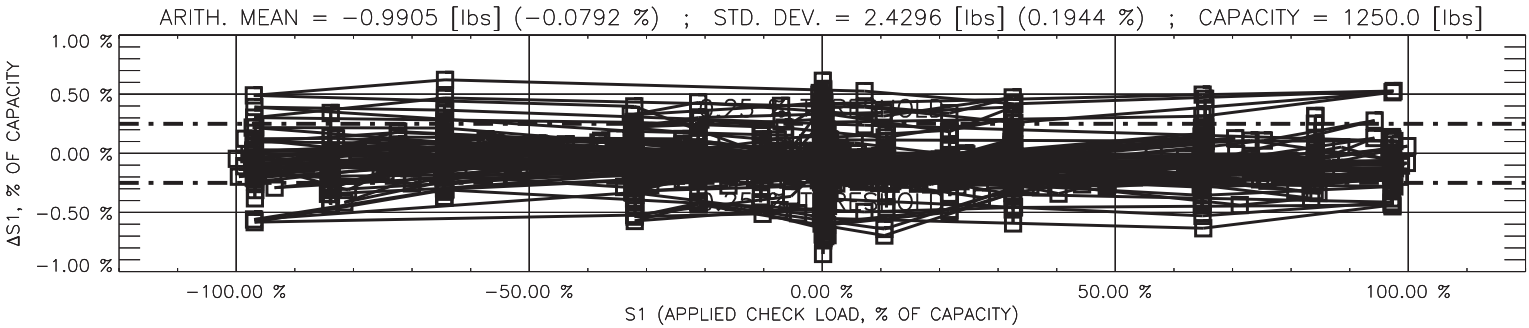

$\Delta \mathrm{S} 2$

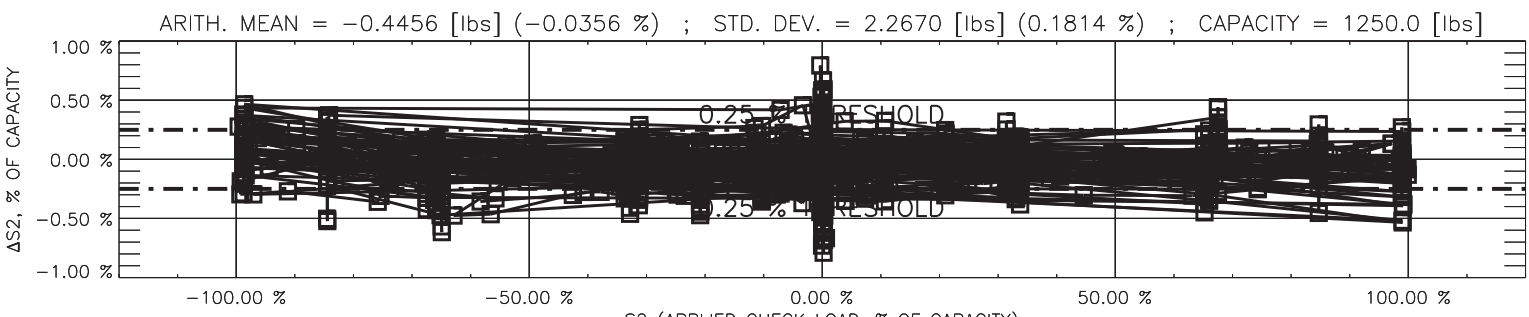

S2 (APPLIED CHECK LOAD, \% OF CAPACITY)
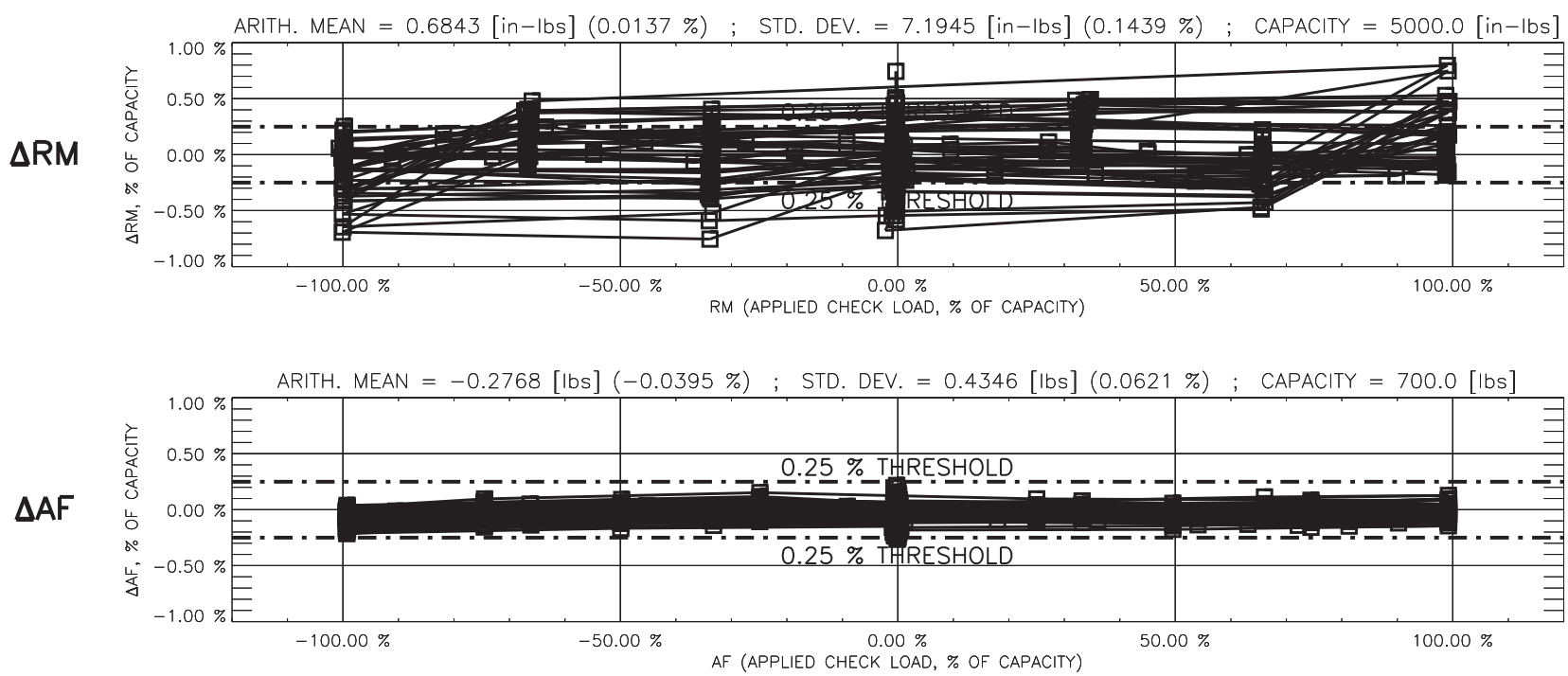

Fig. 13b MC60D: Load residuals of 1906-point data set after application of matrix of 1906-point data set (matrix 2).

American Institute of Aeronautics and Astronautics 


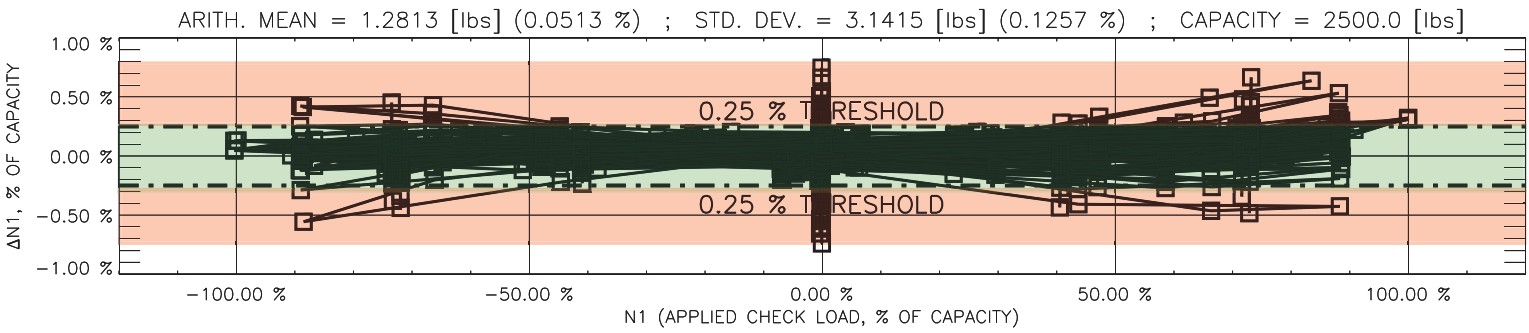

$\Delta \mathrm{N} 2$

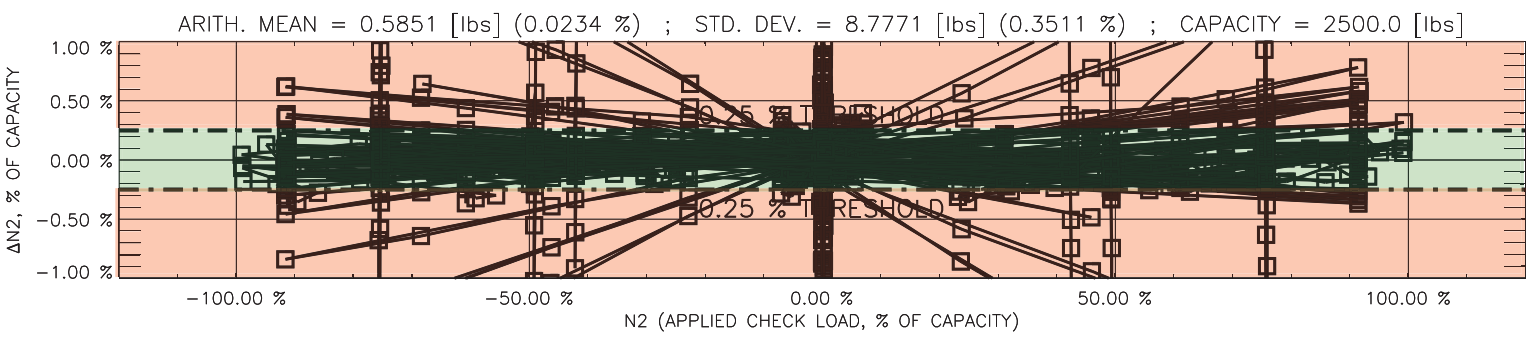

$\Delta \mathrm{S} 1$

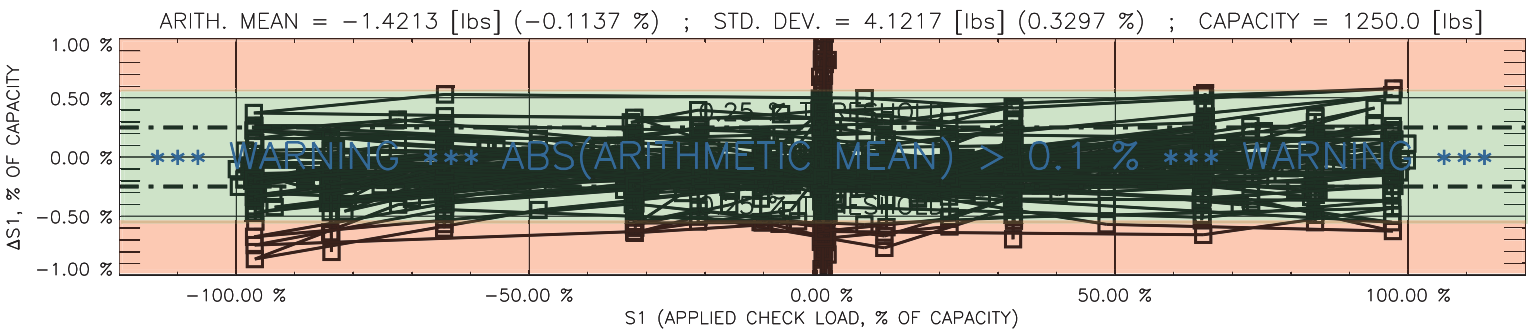

$\Delta \mathrm{S} 2$

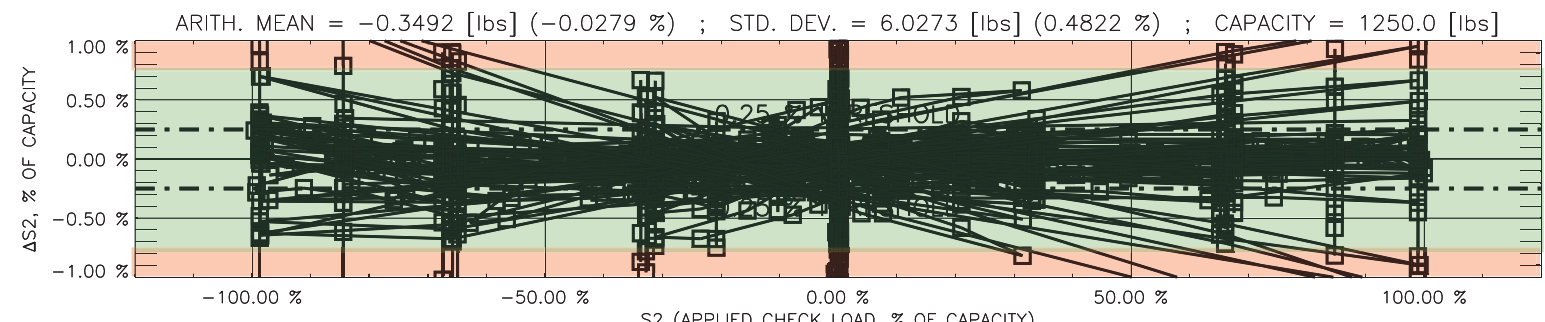

S2 (APPLIED CHECK LOAD, \% OF CAPACITY)
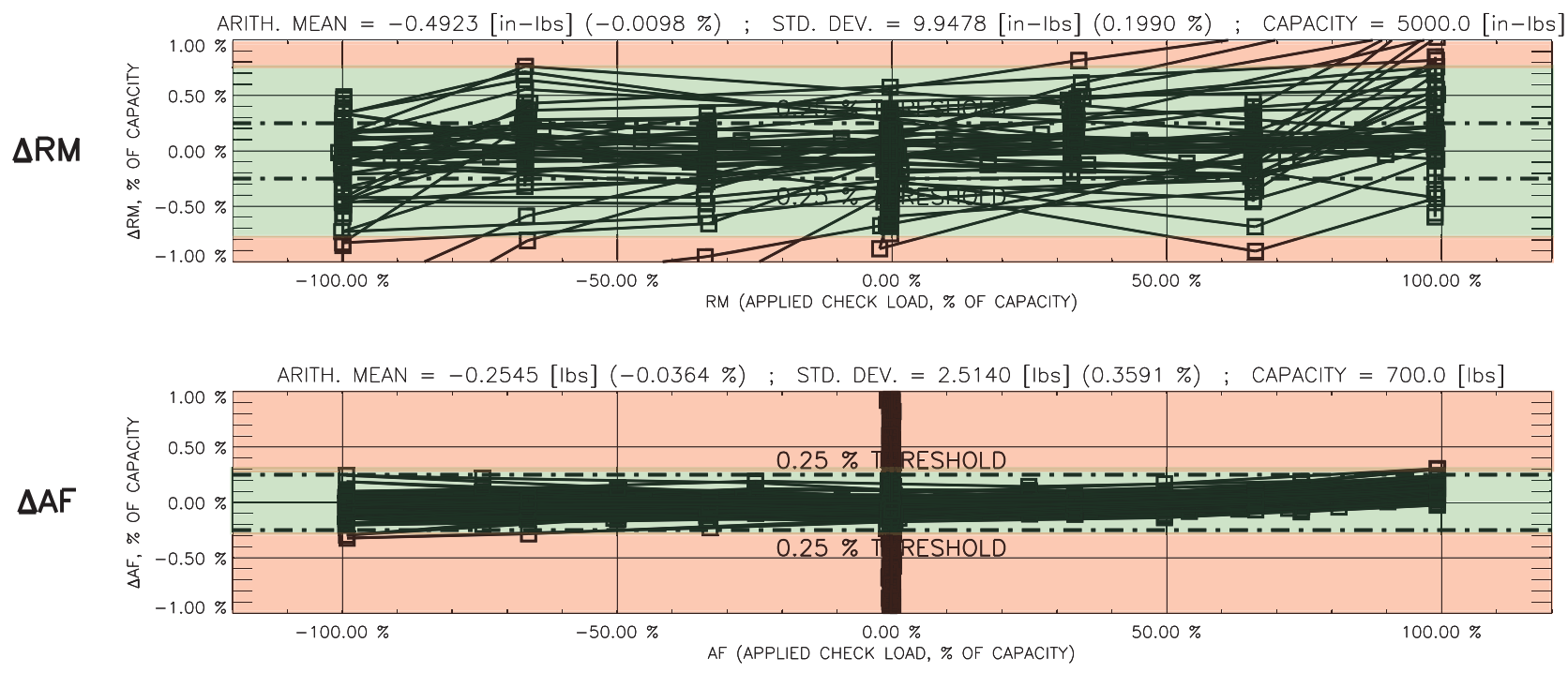

Fig. 13c MC60D: Load residuals of 1906-point data set after application of matrix of 142-point subset (matrix 1). (green region $\equiv$ residuals after use of matrix 2 ; red region $\equiv$ residual increase after use of matrix 1 ) 


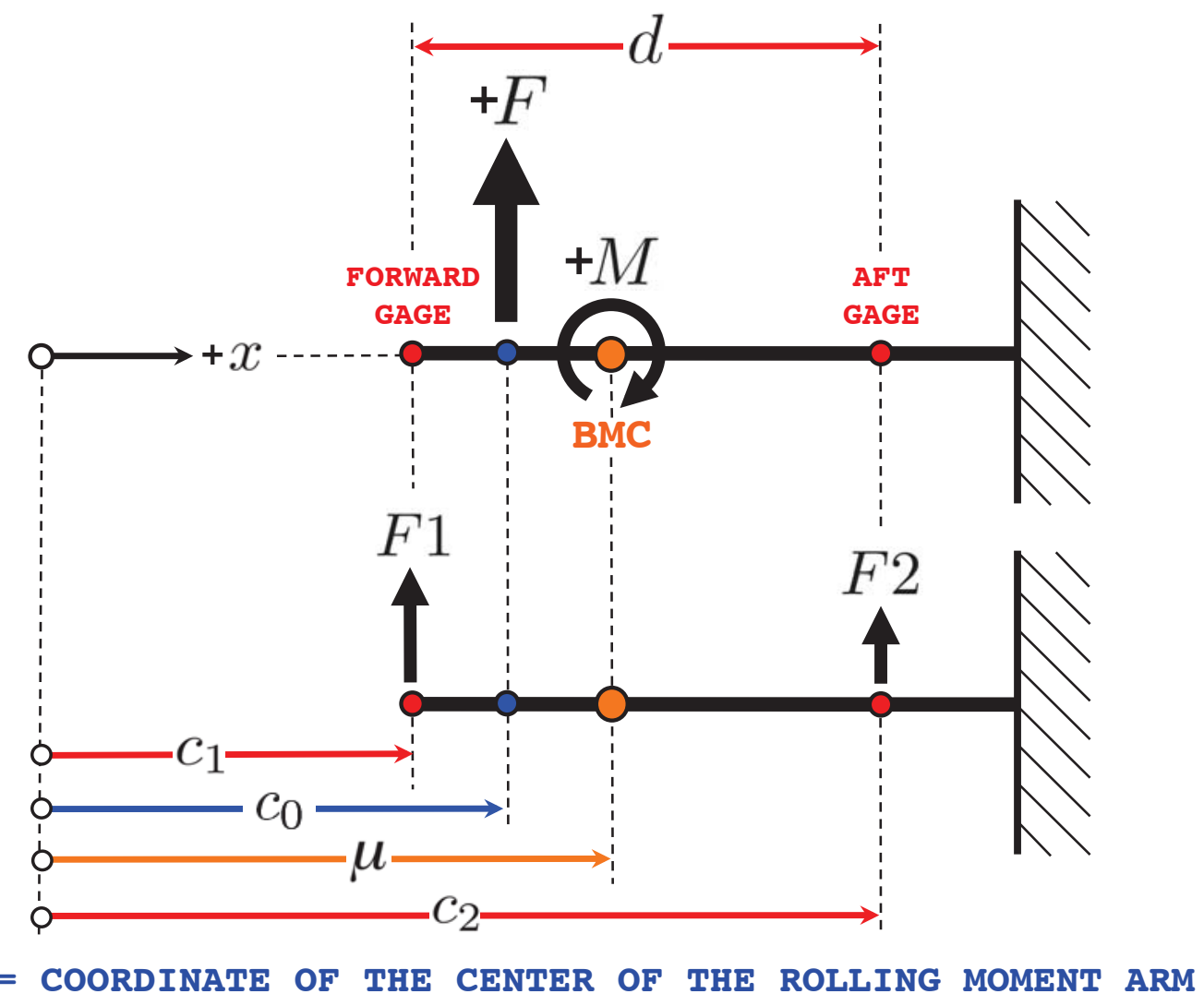

Fig. 14 Calculation of gage load components $F 1$ and $F 2$ as a function of the total force $F$. (balance orientations defined in Fig. 1a determine if the total force $F$ equals $\pm N F$ or $\pm S F$ ) 